\title{
Optical and Electronic Properties of Si Nanoclusters Synthesized in Inverse Micelles
}

\author{
J. P. Wilcoxon, G. A. Samara, and P. N. Provencio \\ Sandia National Laboratories \\ Albuquerque, NM 87185-1421 \\ USA \\ PACS numbers: 82.70.Dd, 71.24.+q, 61.46.+w
}

\begin{abstract}
Highly crystalline, size-selected silicon ( $\mathrm{Si}$ ) nanocrystals in the size range 2-10 $\mathrm{nm}$ were grown in inverse micelles and their optical absorption and photoluminescence (PL) properties were studied. High resolution TEM and electron diffraction results show that these nanocrystals retain their cubic diamond stuctures down to sizes $\sim 4 \mathrm{~nm}$ in diameter, and optical absorption data suggest that this structure and bulk-like properties are retained down to the smallest sizes produced $(\sim 1.8 \mathrm{~nm}$ diameter containing about $150 \mathrm{Si}$ atoms). High pressure liquid chromatography techniques with on-line optical and electrical diagnostics were developed to purify and separate the clusters into pure, monodisperse populations. The optical absorption revealed features associated with both the indirect and direct bandgap transitions, and these transitions exhibited different quantum confinement effects. The indirect bandgap shifts from $1.1 \mathrm{eV}$ in the bulk to $\sim 2.1 \mathrm{eV}$ for nanocrystals $\sim 2 \mathrm{~nm}$ in diameter and the direct transition at $\Gamma\left(\Gamma_{25}-\Gamma_{15}\right)$ blue shifts by $0.4 \mathrm{eV}$ from its $3.4 \mathrm{eV}$ bulk value over the same size range. Tailorable, visible, room temperature $\mathrm{PL}$ in the range $700-350 \mathrm{~nm}(1.8-3.5 \mathrm{eV})$ was observed from these nanocrystals. The most intense PL was in the violet region of the spectrum $(\sim 400 \mathrm{~nm})$ and is attributed to direct electron-hole recombination. Other less intense PL peaks are attributed to surface state and to indirect bandgap recombination. The results are compared to earlier work on Si clusters grown by other techniques and to the predictions of various model calculations. Currently, the wide variations in the theoretical predictions of the various models along with considerable uncertainties in experimental size determination for clusters less than 3-4 nm, make it difficult to select among competing models.
\end{abstract}




\section{DISCLAIMER}

This report was prepared as an account of work sponsored by an agency of the United States Government. Neither the United States Government nor any agency thereof, nor any of their employees, make any warranty, express or implied, or assumes any legal liability or responsibility for the accuracy, completeness, or usefulness of any information, apparatus, product, or process disclosed, or represents that its use would not infringe privately owned rights. Reference herein to any specific commercial product, process, or service by trade name, trademark, manufacturer, or otherwise does not necessarily constitute or imply its endorsement, recommendation, or favoring by the United States Government or any agency thereof. The views and opinions of authors expressed herein do not necessarily state or reflect those of the United States Government or any agency thereof. 


\section{DISCLAIMER}

Portions of this document may be illegible in electronic image products. Images are produced from the best available original document. 


\section{INTRODUCTION}

Because it is an indirect bandgap semiconductor, silicon ( $\mathrm{Si}$ ) has a major drawback: its inability to emit light efficiently, and, furthermore, its weak emission is in the near $\mathbb{R}$. There is presently a large research effort aimed at exploring physical and chemical means to break silicon's lattice symmetry and mix different momentum (k) states in order to induce a useful level of luminescence and optical gain. The approaches include: ${ }^{1}$ (1) impurity-induced luminescence (e.g., S, B, Be, Er), (2) alloy-induced luminescence (e.g., Si-Ge-C), (3) porous silicon, and (4) quantum wires and dots (or nano-size clusters). The first two of these approaches are plagued by, among other things, relatively low luminescence intensity at low temperature which becomes vanishingly weak at room temperature, whereas the last two, which may be mechanistically related via quantum confinement, have considerable potential but have remained largely uncontrolled and poorly understood. Success in this endeavor is obviously a major challenge to materials science, one that could have profound technological implications.

Because visible photoluminescence (PL) has been observed from Si nanoclusters ${ }^{1}$, these clusters and their potential are a subject of current interest. Si nanoclusters have been produced by aerosol techniques, ${ }^{2}$ plasma deposition, ${ }^{3}$ sputtering, ${ }^{4}$ spark ablation ${ }^{5}$ and grown as colloids, ${ }^{6}$ or in glass matrices by a variety of approaches ${ }^{7-9}$ including ion implantation ${ }^{9}$ followed by high temperature annealing; however, all of these techniques produce a large distribution of cluster sizes resulting in very broad optical absorption and PL features which limit usefulness and make definitive interpretation in terms of quantum confinement and other mechanisms difficult.

To understand the origin of visible PL and other electronic properties of Si nanoclusters, it is necessary to study size-selected nanoclusters and to assess the role of surface recombination. Definitive experimental results will be key to future scientific progress and practical utilization of this material. From a physics perspective, such studies should lead to a better understanding of quantum confinement of electrons and holes in indirect bandgap semiconductors. Quantum confinement in direct gap semiconductors such as $\mathrm{GaAs}$ and $\mathrm{CdSe}$ is fairly well understood, but much less is known about confinement in indirect gap materials. ${ }^{10-11}$ The bulk excitonic radius for $\mathrm{Si}$ is $\sim 4 \mathrm{~nm}$ which suggests that quantum confinement effects should be observed for nanocrystals smaller than this size. 
We have developed a synthesis method based on using inverse micelles as reaction vessels ${ }^{12}$ to produce useful quantities of size-selected clusters and have used this method to synthesize a variety of metal and compound semiconductor clusters. ${ }^{13-15}$ These clusters have been remarkable in their size monodispersity and the sharpness and richness of their spectral features which have demonstrated strong quantum confinement effects. In this paper we apply our inverse micellar synthesis method to produce size-selected Si nanoclusters and to study their size-dependent optical absorption and photoluminescence (PL). We shall first discuss the synthesis and characterization of these nanoclusters and then present and discuss their optical properties including comparison with earlier work and theoretical predictions.

\section{SYNTHESIS AND CHARACTERIZATION}

\section{A. Synthesis}

Size-selected nanosize Si clusters were grown by a generic process which is described in detail elsewhere. ${ }^{12.13}$ Controlled nucleation and growth of the nanoclusters occurs in the interior of nanosize surfactant aggregates called inverse micelles. In our process, an anhyrous ionic salt (e.g., $\mathrm{SiX}_{4}$, where $\mathrm{X}=\mathrm{Cl}, \mathrm{Br}$, or $\mathrm{I}$ ) is dissolved in the hydrophilic interior of a solution of micelles. The basic idea is that since the ionic salts used are completely insoluble in the continuous oil medium used (e.g. octane), nucleation and growth of $\mathrm{Si}$ is restricted to the micelle interior. The interior volume of micelles can be varied over the range of $1-10 \mathrm{~nm}$. We emphasize that the anhydrous salt is dissolved to form a transparent ionic solution but with a complete absence of water; in a sense the salt is "hydrated" by the micelle. The absence of water prevents simple hydrolysis to form $\mathrm{SiO}_{2}$ which is why this synthesis must be performed in waterfree oils like octane or decane, and using a controlled atmosphere glove box. Similarly, the surfactants used, both nonionic aliphatic polyethers, or alternatively quaternary ammonium cationic surfactants, must be dissolved in anhydrous THF and dried over Na metal to remove any small traces of water. Alternatively, the surfactants may be purified by HPLC, which results in even better quality, but is more tedious. Once the $\mathrm{Si}$ halide salts are solubilized in the inverse micelles, the formerly clear inverse micelle solution takes on the color of the salt (clear for the $\mathrm{Cl}$ 
salts, light yellow for the $\mathrm{Br}$ and I types). This transparent precursor solution has distinct absorbance peaks much as do charge transfer complexes of other metal salts (e.g. $\mathrm{CoCl}_{2}$ ) in water.

We next reduce $\mathrm{Si}(\mathrm{IV})$ to $\mathrm{Si}(0)$ using an anhydrous metal hydride, (usually $1 \mathrm{M} \mathrm{LiAlH}_{4}$ in THF). The reduction is rapid with vigorous bubbling as $\mathrm{H}_{2}$ gas is released, electrons are transferred to the $\mathrm{Si}(\mathrm{IV})$ and the light yellow solution becomes clear (for the smallest clusters formed). One can determine the correct stoicheometry of the reaction by following the disappearance of the Si(IV) charge transfer peaks from the precursor solution. Generally we find that for complete reduction we need to use a 2 -fold excess of the $\mathrm{LiAlH}_{4}$ reducing agent. Altematively, it is possible to effect a 4-electron transfer reaction to $\mathrm{Si}(\mathrm{IV})$ using alkaline $\mathrm{N}_{2} \mathrm{H}_{4}$, but there is a possibility of competing hydrolysis to $\mathrm{SiO}_{2}$, because of the presence of water. Our experience is that this hydrolysis is actually slower than the reduction, however, so either reducing agent is acceptable.

Control over the final cluster size is achieved by variation of the micelle size, intermicellar interactions (e.g., proximity to phase boundaries which determines micelle diffusion rates) and reaction chemistry. Size-selected Si clusters with diameters between 1.8 and $\sim 10 \mathrm{~nm}$ were produced. Spectroscopy and high pressure liquid chromatography (HPLC) with on-line spectroscopy, conductivity and refractive index diagnostics were used to demonstrate $100 \%$ reduction of the $\mathrm{Si}(\mathrm{IV})$ to the final $\mathrm{Si}(0)$ nanocluster form. All reactions took place in anaerobic conditions in a Vacuum Atmospheres dry box in which continuous oxygen and moisture removal are monitored by appropriate sensors. Typical oxygen levels were 0.1 to $1 \mathrm{ppm}$ and moisture levels were 0.5 to $3 \mathrm{ppm}$ in the dry box. All solvents and surfactants used were HPLC grade and were completely dust free. The latter is critical to prevent inhomogeneous nucleation.

Since there is no source of oxygen in the reaction mixture, and anhydrous metal hydrides are used as reducing agents, it is possible that the Si cluster surface is terminated by hydrogen from the metal hydride, although we currently have no direct proof of this. When kept in the glove box under Ar, there appears to be no long term (i.e. 6 month to 1 year) degradation of the Si nanoclusters. However, upon exposure to oxygen, a yellowing of the solution occurs which, sometimes over a period of weeks, leads to an observable precipitate. It is possible that oxidation of the surface renders the nanocluster surface hydrophilic, and since the clusters are in a solution 
of very hydrophobic oils, they simply lose their solubility. A hydrogen terminated Si surface, on the other hand, is hydrophobic and likely more stable in the oil.

\section{B. Chromatography}

HPLC separation and elution peak spectral data were obtained using two apparati; (1) a HP model 1050 HPLC system, with automated fraction collection, complete solvent purging, and three sequential detectors, a photodiode array spectrometer (for absorbance measurements between 180 and $600 \mathrm{~nm}$ ), a PL detector (with selectable excitation wavelength and full scanning capability between 200 and $800 \mathrm{~nm}$ ), and a refractive index detector; (2) a Waters HPLC system with a photodiode array spectrometer with a range of 190-800 nm, a conductance detector, and a refractive index detector. The fraction collector developed by us allowed fractions to be collected without exposure to air or moisture. It consists of a programmable microvalve and small teflon solvent lines which are filled with Ar-purged solvent up to a needle which penetrates a septum on a collection vial filled with Ar. Under computer control, the valve switches from the waste position to the sample position (up to six fractions are possible), at the time interval corresponding to the elution of the desired nanocluster fraction. Since the sample is extracted from sealed, crimp-type, sampling vials filled in the glove box, the complete HPLC operation and/or fraction collection can be both moisture- and air-free. In addition, the versatility of our fraction collection scheme allows samples to be collected in septum-sealed quartz cuvettes filled with Argon and subsequently studied with conventional fluorescence and UV-visible spectrophotometers.

Previous HPLC experiments on $\mathrm{Si} / \mathrm{SiO}_{2}$ nanoclusters synthesized in the gas phase showed

a broad range of elution times ${ }^{2,10}$ which is in contrast with our results where the Si peak is of comparable width to that from the other molecular constituents of the solution. Figure 1 shows an example of using chemical affinity chromatography to separate nanosize $\mathrm{Si}$ from other chemicals in the reaction bath. The clusters were stabilized with octylamine. The excess (i.e. unbound) octylamine separates from the clusters as do the surfactant and solvent used in the synthesis, leaving the clusters dispersed in anhydrous acetonitrile (ACN). The complete absorbance spectrum of the clusters and the PL could also be measured during the chromatography and will be shown later. We note that the elution peak width of these $\mathrm{Si}$ 
nanoclusters (size $=2.2 \mathrm{~nm}$ ) is comparable to the completely monodisperse chemical octylamine, showing very little size dispersion.

Fortunately, by using organically coated Si columns we were able to use a simple, one component organic mobile phase in our work, since it turned out that our Si nanoclusters were uncharged. However, to discover the best conditions for this chromatography was quite difficult and highly empirical. These conditions were also quite different from other nanoclusters we have studied by HPLC. ${ }^{15}$

There are three valuable features of HPLC which helped us determine optimal synthesis conditions. (1) Any unreacted salt elutes as a separate peak (usually at the same time as the surfactant), (2) any charged species (e.g. salt, ionic surfactants) can be identified by using an online conductivity meter, and (3) nanoclusters of different sizes can be separated and their optical properties determined on-line, without exposure to oxygen. The second feature of HPLC analysis is illustrated in Fig. 2 by the separation of Si clusters made using a charged cationic surfactant. We observed that the ionic byproducts of the reaction and the ionic surfactant are separated in time from the small neutral Si nanoclusters.

Finally, we illustrate separation of two sizes of Si nanoclusters in Fig 3. Using the PL detector we can identify which size of cluster has significant room temperature PL, and also make sure that no impurity organic chemicals could be giving rise to the PL signal. Only the absorbance peak corresponding to the more numerous population of small, $2.0 \mathrm{~nm}$ clusters coincides with a strong visible PL signal. We also obtained the complete absorbance and PL wavelength dependences for each size Si nanoclusters as will be shown in Sec. III. Inductively coupled plasma/mass spectroscopy (ICP-MS) of the collected Si fractions showed that nearly $80 \%$ of the total $\mathrm{Si}$ was recovered by HPLC, and the only inorganic detected was $\mathrm{Si}$. Gas chromatography/mass spectroscopy (GC/MS) showed the only significant organic chemical in the collected fractions was the mobile phase solvent (i.e. the fractions had been chemically purified as desired). In particular, no surfactant was detected.

\section{Other Characterization}

In addition to the on-line HPLC optical and size characterization discussed above, we used x-ray diffraction (XRD), selected area electron diffraction (SAD), and high-resolution 
transmission electron microscopy (HRTEM) to examine the Si nanoclusters. HRTEM lattice fringe images of $d=2 \mathrm{~nm}$ and $d=8-10 \mathrm{~nm}$ Si clusters shown in Fig. 4 reveal the high crystalline quality of these clusters. Since the contrast between the Si and the amorphous holey carbon grid on which the clusters are deposited from solution is low, there is $10 \%$ uncertainty in determining the edges (size) of the clusters (this is only one lattice plane in the case of the smallest clusters).

The largest clusters $(d=8-10 \mathrm{~nm})$ were collected as a powder after HPLC purification and subjected to XRD analysis. The diffraction lines are identical to those of bulk Si (Fig. 5). Interestingly, no oxide diffraction peaks were observed, though it was not possible to exclude air during the XRD measurement. The SAD reflections on these clusters were also the same as the bulk, though broadened somewhat by the finite crystallite size. A SAD pattem is shown in Fig. 6 where the innermost ring corresponds to the $<111>$ orientation with a d spacing of $3.1 \AA$ as in bulk Si.

\section{RESULTS AND DISCUSSIONS}

\section{A. Optical Absorption}

\section{Bulk Absorption}

In order to better understand the optical absorption spectra of Si clusters, it is instructive to review briefly the main features of the spectrum of bulk $\mathrm{Si}^{16}{ }^{16}$ These features, which are shown in Fig. 7, reflect the details of the band structure ${ }^{17}$ shown in Fig. 8. The long absorption tail between 1.2 and $\sim 3 \mathrm{eV}$ reflects the indirect nature of the bandgap. The sharp rise in absorption with increasing photon energy starting around $3.2 \mathrm{eV}(380 \mathrm{~nm})$ is associated with the direct transition at the $\Gamma$ point $\left[\Gamma_{25} \rightarrow \Gamma_{15}\right]$ whose energy is $3.4 \mathrm{eV}(365 \mathrm{~nm})$, and the second sharp rise starting around $4 \mathrm{eV}(320 \mathrm{~nm})$ is associated with a second direct transition, most likely the $\Gamma_{25}-$ $\Gamma_{2^{\prime}}$ transition whose energy is $4.2 \mathrm{eV}(295 \mathrm{~nm})$ or possibly the direct transition at X. 


\section{Nanocrystal Spectra}

The measured optical response (or extinction) of nanocrystals reflects the sum of the scattering and absorption. The scattering needs to be taken into consideration in comparing cluster spectra to that of bulk $\mathrm{Si}$.

Figure 9 shows the extinction spectrum of relatively large $(d=10 \mathrm{~nm})$ Si clusters calculated ${ }^{2,10}$ from Mie theory and compares it to the absorption spectrum of bulk Si. The two spectra are normalized around the absorption shoulder corresponding to the $\Gamma_{25} \rightarrow \Gamma_{15}$ direct transition. One of the obvious differences in the two spectra is the significantly enhanced extinction of the clusters which is due to scattering. Shown also in Fig. 9 are three cluster spectra (also normalized at about the shoulder of the $\Gamma_{25} \rightarrow \Gamma_{15}$ transition), two ( $d=10$ and $1.8 \mathrm{~nm}$ ) from

the present work and the third ( $d=3.7 \mathrm{~nm}$ ) from Kanemitsu's work. ${ }^{8}$ Our $d=10 \mathrm{~nm}$ spectrum is from one of our earliest samples that was not purified and size-separated by HPLC, and thus we suspect that it had a relatively broad size distribution. Because of uncertainties in the absolute values of the measured extinction coefficients, the normalization of the nanocrystal spectra in Fig. 9 is only approximate and is merely intended to reveal the shape and influence of scattering, leaving the discussion of the details of the cluster spectra until later.

Some of our Si nanocrystal samples exhibited considerable structure in their absorption spectra while others had less structured spectra. The difference is undoubtedly related to the details and subtleties of the synthesis, but the reason is not known at present. Broad distributions of nanocrystal sizes can of course smear out spectral features, and impurities and unreacted precursors can be responsible for extrinsic spectral effects. Our HPLC separation procedures should rule out both effects, and, as will be shown, most of the observed features appear to be intrinsic to Si nanocrystals.

Figure 10 shows the absorption spectra of two of our nanocrystal samples (curves 1 and 3) with emphasis on the behavior at long wavelengths, i.e., in the indirect band gap tail region. Curve 1 is for the same $d=10 \mathrm{~nm}$ sample (curve 3) in Fig. 9. The shoulder at $\sim 370 \mathrm{~nm}$ is attributed to the $\Gamma_{25} \rightarrow \Gamma_{15}$ direct gap. Curves 3 is featureless over the range of the data shown. Similar featureless spectra have been observed by other workers on Si nanocrystals embedded in glass matrices or from surface oxidized nanocrystals. However, subtle features are sometimes observed. Littau et al's results ${ }^{2}$ on the larger $(d=6.5 \mathrm{~nm})$ of their two samples exhibit a weak 
hint of the direct-gap absorptions at $-360 \mathrm{~nm}$ (curve 2 in Fig. 10) and at $\sim 290 \mathrm{~nm}$ (not shown). Similarly, there is an inflection point in the absorbance in Kanemitsu's data ${ }^{8}$ at $\sim 350 \mathrm{~nm}$ (curve 4). We have seen this feature (often much more pronounced as in curve 1) in the absorbance data on several of our samples. A particularly interesting example for $d=8-10 \mathrm{~nm}$ nanocrystals is shown in Fig. 11 where the feature appears as a shoulder at $\sim 370 \mathrm{~nm}$ followed by a relatively sharp increase in absorbance and double peaks at $\sim 270 \mathrm{~nm}$ and $220 \mathrm{~nm}$. The resemblance of these features and closeness in $\lambda$ to those in the spectrum of bulk Si (see Fig. 7) is striking indicating that clusters of this size $(d=8-10 \mathrm{~nm})$, which is comparable to the size of the excitonic diameter in the bulk, retain much of the character of bulk Si with very little evidence for quantum confinement effects.

Some of our smaller size nanocrystal samples exhibited highly structured absorption spectra. An example is shown in Fig. 12 for a sample with $d=2.0 \mathrm{~nm}$. The figure also shows the bulk Si spectrum for comparison. The two spectra are remarkably similar with that of the nanoclusters shifted to shorter (higher) wavelength (energy). The long absorption tail associated with the indirect bandgap and the two direct transitions $\left(\Gamma_{25} \rightarrow \Gamma_{15}\right.$ and $\Gamma_{25} \rightarrow \Gamma_{2}$ or possibly that at X) are very well defined in the nanocrystal spectrum. Because our samples are very dilute $\left(\sim 10^{-4} \mathrm{molar}\right)$ the signal-to-noise ratio for this sample is low for the low absorbance associated with the indirect transitions, hence the noise in this region of the spectrum. For other samples, the signal-to-noise was considerably higher and the spectrum less noisy in this region.

Figure 13 provides a linear absorbance vs. wavelength plot for the same two samples as in Fig. 12 with emphasis on the region of the direct transitions. The absorbances have been normalized as before to the value at the shoulder associated with the $\Gamma_{25} \rightarrow \Gamma_{15}$ transition. The close resemblance of the two spectra is remarkable indicating that the bulk-like character of the band structure of $\mathrm{Si}$ is preserved down to the $d \approx 2 \mathrm{~nm}$ size (i.e. down to nanocrystals with 200 atoms or less). The data in Fig. 13 show clear evidence for quantum confinement; specifically, both direct transitions of the nanocrystals are blue-shifted by about $0.4 \mathrm{eV}$ compared to the bulk. As we shall see below, this is a smaller quantum confinement effect than is found for the indirect gap, but it is larger than is predicted by model calculations.

There is another aspect of the results in Fig. 13 that deserves attention. Reference to Fig. 8 reminds us that the $\mathrm{Si}$ conduction band at $\Gamma$ involves two overlapping bands of $\mathrm{p}_{\sigma *}$ states, one 
with positive and the other with negative dispersion. Quantum size confinement can be expected to influence these two bands differently resulting in their splitting which in turn might be reflected in the shape of the $\Gamma_{25} \rightarrow \Gamma_{15}$ absorption. While model calculations ${ }^{18}$ do indeed predict such splitting, our cluster results in Fig. 13 and for other samples do not reveal it. However, our result ${ }^{19}$ and those of others ${ }^{20}$ on Ge nanocrystals do show evidence for such splitting.

The absorption data in Figs. 10 and 12 suggest a blue shift of the indirect absorption tail with decreasing nanocrystal size, and the gap appears to remain indirect. The small absorbance values in the tail region along with some uncertainty due to correcting the data for the scattering contribution make detailed analysis of the data in this wavelength region uncertain. However, the quality of the data on several of our samples was sufficiently good to allow meaningful analysis. For an indirect transition the absorption data in the region of the band edge can be described by ${ }^{21}$

$$
\alpha h v=C\left(h v-E_{g}\right)^{2},
$$

where $\alpha$ is the absorption coefficient, $h v$ is the photon energy, $\mathrm{C}$ is a constant and $\mathrm{E}_{\mathrm{g}}$ is the bandgap energy. Absorption data on bulk Si can be fit by this relationship, and Fig. 14 shows that it also holds for $\mathrm{Si}$ nanoclusters. In the figure $(\alpha h v)^{1 / 2}$ is plotted vs. $h v$ for $d=1.8 \mathrm{~nm}$ clusters. The intercept of the linear response with the $\mathrm{x}$-axis is the indirect bandgap. These results yield $E_{g}=2.2 \mathrm{eV}$. While the uncertainty in this value of $E_{z}$ may be as large as $\pm(0.2-$ $0.3) \mathrm{eV}$ because of the aforementioned weak absorption of the cluster solutions in the tail region as well as some inaccuracies introduced in correcting for scattering, the result clearly demonstrates a significant quantum confinement effect for the indirect gap of $\mathrm{Si}$. Our result is in close agreement with a study by Brus et $\mathrm{al}^{2 \mathrm{~b}}$ on $\mathrm{SiO}_{2}$-capped $\mathrm{Si}$ nanocrystals. From photoluminescence excitation (PLE) and photoluminescence (PL) measurements on nanoclusters estimated to have a Si core diameter in the range 1-2 nm, these authors deduced a bandgap of $2.06 \mathrm{eV}$. As we shall discuss later, these measured quantum confinement effects are smaller than those predicted by effective mass theory but are comparable to results of some model calculations. 


\section{Optical Absorption of Very Small Si Nanocrystals}

Our smallest $\mathrm{Si}$ nanocrystals $(d=1.8 \mathrm{~nm})$ have $\sim 150$ atoms and still retain bulk-like optical properties. There has been considerable theoretical interest in the structure, shape and properties of $\mathrm{Si}$ clusters containing up to few tens atoms and in answering the question of how small can Si clusters be and still retain bulk-like properties. ${ }^{22-24}$ The results have revealed major changes in shape as a function of size with a qualitative change from prolate to more spherical structures in the narrow range between 24 and 30 atoms. $^{22}$

Rinnen and Mandich ${ }^{25}$ determined the absorption spectra of gas-phase neutral Si clusters in the size range $\mathrm{Si}_{18}$ to $\mathrm{Si}_{41}$ using resonant one-and two-color photodissociation spectroscopy. A surprising aspect of their results is that the spectra are essentially identical over the whole size range studied, despite the theoretical findings that there is a wide variation in the structure of the clusters over this range. The spectrum of $\mathrm{Si}_{30}$ is shown and compared to bulk and $d=2 \mathrm{~nm}$ cluster spectra in Fig. 13. The authors observed that the $\mathrm{Si}_{18-41}$ spectra have much in common with the spectrum of bulk crystalline $\mathrm{Si}$; however, the similarity in the spectra is not so obvious.

On the basis of their results Rinnen and Mandich ${ }^{25}$ concluded that these small Si clusters must share one or more common structural entities which are strong chromophores. One possibility is that these small clusters share a common bonding network which persists and extends as the cluster grows in size, and that this network may be related to that of bulk Si (as suggested by their conclusion that the spectra of their small clusters and bulk $\mathrm{Si}$ are similar). Another possibility is that all of these clusters contain at least one loosely bound smaller cluster such as the abundant $\mathrm{Si}_{10}$ which may be responsible for the sharp spectral structure in Fig. 13. We believe that the latter explanation is the correct one and offer the following explanation.

Rinnen and Mandich estimated that in their photodissociation experiments the temperature of the clusters was in the range $700-900 \mathrm{~K}$, i.e., their spectra are high temperature spectra. In work on metallic and semiconducting clusters, it is generally found that the melting point is substantially depressed with decreasing cluster size. Thus, for example, the melting point of CdSe decreases sharply with decreasing cluster size, its value for $d=3 \mathrm{~nm}$ being about one half of its bulk value and is expected to be much less for smaller clusters. ${ }^{11}$ Thus, we suggest that for the conditions of Rinnen and Mandich's experiments, their Si clusters are essentially in a "molten state" and that small entities like $\mathrm{Si}_{10}$ or smaller are indeed the dominant 
species. These entities have closed shape configurations ${ }^{26}$ which can quite conceivably exist in a loosely-bound, liquid-like state. We shall return below to a discussion of the spectra of small $\mathrm{Si}$ nanocrystals and to a comparison of our results to theory.

\section{B. Photoluminesence}

\section{General Considerations}

The fate of photogenerated electron-hole pairs in a semiconductor is determined by traps and intrinsic recombination processes. As noted earlier, weak light emission, or photoluminescence (PL), from phonon-assisted, indirect bandgap $e-h$ recombination in bulk $\mathrm{Si}$ has been observed. ${ }^{27}$ At room temperature, this PL peaks in the near $\mathbb{R}$ at $1130 \mathrm{~nm}(=1.1 \mathrm{eV})$, and at low temperatures (e.g., 77K) there is also emission from, or to, shallow impurity levels. In the present work on Si nanocrystals we have observed room temperature emission at various wavelengths in the range $700-350 \mathrm{~nm}(\sim 1.8-3.5 \mathrm{eV})$, i.e., across the visible range. All our data were obtained at room temperature (295K) and the solvent was in almost all cases acetonitrile.

In small nanocrystals, a large number of the atoms are at or near the surface leading to a preponderance of dangling bonds and defects which result in surface states. Adsorbed impurities can produce additional surface states, and all of these states can act as traps or recombination sites. Consequently, light emission from nanostructures, be they nanocrystals or porous $\mathrm{Si}$, can be quite complex with considerable uncertainties about the origin of the observed PL. In solution-grown nanocrystals such as our own, there is the added concern that excess precursors and reaction products can lead to some luminescence which can interfere with that due to the $\mathrm{Si}$ clusters. In this regard, chromatographic separation/purification has led to significant improvement in the quality of the observed PL spectra. Even so, it is difficult to definitively rule out the role of contaminants in the measured PL spectra of nanoclusters in colloidal solutions.

In considering optical absorption and PL spectra it is necessary to take into account the exciton binding energy, $E_{B}$, when the PL is excitonic in nature. The excitonic transition energy $E_{e x}$ is given by ${ }^{16}$ 


$$
\begin{aligned}
E_{e x} & =E_{g}-E_{B} \\
& =E_{g}-\frac{\mu e^{4}}{2 \hbar^{2} \epsilon^{2} n^{2}},
\end{aligned}
$$

where $\mu$ is the reduced mass of the exciton which is related to the electron and hole effective masses by $\mu^{-1}=m_{e}^{-1}+m_{h}^{-1} ; e$ is the electronic charge, $\epsilon$ is the dielectric constant and $n$ is the principal quantum number. The exciton binding energy for nanocrystals is expected to be significantly enhanced over its bulk value due to quantum confinement and the expected smaller $\epsilon$ of the nanocrystals. For example, Zunger and $\mathrm{Wang}^{28}$ find $\epsilon \approx 8$ for $d=2 \mathrm{~nm}$ Si nanocrystals compared with a bulk value of 11.4. The smaller $\epsilon$ also strengthens the Coulombic $e-h$ attraction which is given by $U(r)=-e^{2} / \epsilon r$, where $r$ is the distance between the electron and hole.

Tight binding calculations by Leung and Whalley ${ }^{29}$ yield a large increase in $E_{B}$ of $\mathrm{Si}$ with decreasing cluster size. Similarly, Takagahara and Takeda ${ }^{30}$ calculated a large increase in $E_{B}$ with decreasing cluster size for indirect-gap semiconductors in the framework of effective mass theory. Their results for Si show that $E_{B}$ increases from $14.3 \mathrm{meV}$ for the bulk to $\sim 250$ $\mathrm{meV}$ for $d=2 \mathrm{~nm}$ clusters. The large enhancements in $E_{B}$ for Si nanocrystals makes it likely that luminescence from them, even at room temperature, is due to localized excitons.

\section{Results on Nanocrystals}

Figure 15 shows a room temperature PL spectrum of one of our earliest nanocrystal samples ( $d \leq 5 \mathrm{~nm}$ ) obtained before developing our chromatographic separation/purification capability. For excitation at $256 \mathrm{~nm}$, the PL spectrum exhibits intense, structured emission centered at $\sim 365 \mathrm{~nm}(3.4 \mathrm{eV})$ and a weaker, also structured, emission centered at $600 \mathrm{~nm}(2.06$ $\mathrm{eV}$ ). It may well be that the structure in both emissions is associated with the presence of impurities or even different size populations. We believe that the $365 \mathrm{~nm}$ emissions is largely due to direct $e-h$ recombination at $\Gamma\left(\Gamma_{25}-\Gamma_{15}\right)$, and its high intensity and short PL decay lifetime ( $\tau=1.5 \mathrm{~ns}$, as will be discussed later) are consistent with this assignment. The origin of 
the $600 \mathrm{~nm}$ PL is more uncertain. If both emissions were intrinsic properties of Si nanocrystals, then the $600 \mathrm{~nm}$ emission would be due to bandgap recombination. In this scenario, reference to the absorption data discussed above, would suggest that the 365 and $600 \mathrm{~nm}$ emission peaks are associated with a small nanocrystal population with $d \cong 2 \mathrm{~nm}$.

The dashed curve in Fig. 15 is the PL spectrum of $\mathrm{SiO}_{2}$-capped Si nanoclusters reported by Littau et al ${ }^{2 a}$ for their " 1.0 colloid" sample $(d<5 \mathrm{~nm})$ excited at $350 \mathrm{~nm}$. We believe that this luminescence has the same origin as that peaked at $\sim 600 \mathrm{~nm}$ in our spectrum, but is red shifted because of larger crystallite size. As will be discussed later, we believe this luminescence is due to bandgap indirect recombination.

For excitation at $320 \mathrm{~nm}$ the PL spectrum for our sample in Fig. 15 retains its structured two-peak character, but the peaks are red shifted compared to excitation at $256 \mathrm{~nm}$. Specifically, the more intense emission is centered at $\sim 380 \mathrm{~nm}(3.3 \mathrm{eV})$ and the less intense emission is peaked at $\sim 680 \mathrm{~nm}(1.82 \mathrm{eV})$. The red shift of the emission may come from exciting a population of larger clusters with the longer wavelength excitation. The $-680 \mathrm{~nm}$ peak in our PL spectrum excited at $320 \mathrm{~nm}$ is red shifted from Littau et al's $\mathrm{s}^{2}$ PL spectrum in Fig. 15, consistent with a larger cluster population.

Results on a $d=2 \mathrm{~nm}$ size-selected, purified sample are shown in Fig. 16. The absorption spectrum was discussed earlier (Figs. 12 and 13). The first absorption peak at $325 \mathrm{~nm}$ $(3.81 \mathrm{eV})$ is attributed to the $\Gamma_{25}-\Gamma_{15}$ direct gap, but blue shifted by $-0.4 \mathrm{eV}$ due to quantum confinement. Excitation at $245 \mathrm{~nm}$ yields the PL spectrum shown. The major peak is centered at $365 \mathrm{~nm}(=3.40 \mathrm{eV})$ i.e., essentially at the same wavelength as for the sample in Fig. 15. Again, we attribute this peak to direct $e-h$ recombination at $\Gamma$. It is red shifted from the absorption peak by $0.4 \mathrm{eV}$ due to the strong exciton binding energy for very small clusters, i.e., $E_{B} \approx 0.4$ $\mathrm{eV}$. This large value of $E_{B}$ is comparable to values deduced from model calculations for $\mathrm{Si}$ and is due to quantum confinement and reduced electronic screening. As we have already noted, Takagahara and Takeda ${ }^{30}$ find $E_{B}>0.25 \mathrm{eV}$ for the indirect gap of Si clusters with $d \leq 2 \mathrm{~nm}$. Tagaki et al ${ }^{3}$ deduced a value of $E_{B}=0.32 \mathrm{eV}$ for $d \approx 3.3 \mathrm{~nm}$ Si clusters. Similarly, Read et a ${ }^{31}$ find $E_{B}=0.32 \mathrm{eV}$ for the indirect gap of $\mathrm{Si}$ quantum wires of $\sim 3 \mathrm{~nm}$ diameter. More recent 
first principles calculations by Ögüt et al ${ }^{32}$ show $E_{B}$ increasing from $0.3 \mathrm{eV}$ for $d=3 \mathrm{~nm}$ to $~$ $1.0 \mathrm{eV}$ for $d=1 \mathrm{~nm}$.

It can be seen that the major PL peak in Fig. 16 is asymmetric. Analysis of the spectrum reveals a secondary peak at $\sim 420 \mathrm{~nm}(\sim 3.0 \mathrm{eV})$. As appears to be typical for Si nanostructures, this $365 \mathrm{~nm}$ peak (with the $420 \mathrm{~nm}$ peak subtracted) is fairly broad, its width at half max being $-1 \mathrm{eV}$. This sample also exhibits weaker and quite broad emission at wavelengths longer than $500 \mathrm{~nm}$, as shown. The luminescence is less well defined than that for the sample in Fig. 15. In addition to being purer, the sample in Fig. 16 is also more monodisperse as suggested by its sharper absorption features. Consistent with this observation, the extra structure in the PL spectrum in Fig. 15, attributed to different size populations of nanocrystals, is absent in Fig. 16.

Figure 16 also shows the PL spectrum for a similar sample excited at $490 \mathrm{~nm}(2.53 \mathrm{eV})$ i.e., just above the indirect gap for this nanocrystal size. This PL peak is centered at $580 \mathrm{~nm}$ $(2.14 \mathrm{eV})$, and it is tempting to attribute it to indirect bandgap recombination. However, we note that this luminescence is identical to that observed on much larger $(d \cong 8-10 \mathrm{~nm})$ nanocrystals as shown in Fig. 11. We are thus led to conjecture that this PL is due to surface or defect recombination. The independence of this luminescence of nanocrystal size may be relevant to semiempirical tight-binding and ab initio local density calculations by Allan et $\mathrm{al}^{33}$ which demonstrated the stability of self-trapped excitons at the surface of Si nanocrystals. The excitons are obtained for dimer bonds passivated by, e.g., hydrogen or silicon oxide. Light emission from these trapped excitons is essentially independent of size.

Figure 17 shows the absorption and PL spectra of a nanocrystal sample $(d=4 \mathrm{~nm})$ with structured absorption [peaks at $355 \mathrm{~nm}(3.48 \mathrm{eV})$ and $280 \mathrm{~nm}(4.40 \mathrm{eV})$ which we believe are associated with the quantum confinement-shifted two direct transitions at $\Gamma$ ] exhibited a PL peak centered at $540 \mathrm{~nm}(2.30 \mathrm{eV})$ for excitation at $470 \mathrm{~nm}(2.64 \mathrm{eV})$, i.e., well below the first direct transition at $\Gamma$. This emission is at slightly shorter wavelength than the peaks in Figs. 11 and 16 suggesting extrinsic origin. The PL spectrum in Fig. 18 also has a secondary peak at $700 \mathrm{~nm}(\sim 1.8 \mathrm{eV})$ which is most likely due to recombination at the indirect bandgap. 


\section{Comparison with Earlier Luminescence Results and Discussion}

As we have seen above, $\mathrm{Si}$ nanocrystals in solvents produced by our inverse micellar synthesis photoluminesce at various wavelengths in the range $\sim 700-350 \mathrm{~nm}(\sim 1.8-3.6 \mathrm{eV})$. Fig. 18 summarizes the results for our samples and compares the PL peak energies with those from other authors. In doing this comparison it is necessary to caution that a major uncertainty in the figure is knowledge of the size of the nanocrystals. We have presented the data using the nominal diameters deduced from our work and reported by the various authors. We note that these diameters, often deduced from TEM and TEM fringe images, can be off by a large amount. It is very difficult to be certain that TEM images will give truly representative dimensions and shapes in the absence of large statistical samples. In the few cases where statistical analyses were performed, by others, it was found that nanocrystal samples embody a broad distribution of sizes. These broad distributions are evident to some extent in the very broad PL peaks of nanocrystal Si samples. Even for our HPLC- separated and purified cluster populations, we find relatively broad PL peaks, suggesting, at least in part, size dispersion Undoubtedly, the most accurate size information in Fig. 18 is that due to Schuppler et al. ${ }^{34}$ who deduced the sizes of their $\mathrm{SiO}_{2}$ capped nanocrystals from $\mathrm{Si} K$ near-edge $\mathrm{x}$-ray absorption fine structure (NEXAFS) and extended $\mathrm{x}$-ray absorption fine structure (EXAFS) measurements.

Keeping in mind the above precautions, we can now offer some observations on the data in Fig. 18. Looking first at the results of earlier work, we note that there are differences in the dependence on size reported by different authors as shown by the line segments in Fig. 18 for those cases where results on more than two samples were reported by a given group of authors. Some of the differences, but not all, are almost certainly due to uncertainties in size. Secondly, we note that essentially all of the PL peaks energies on $\mathrm{Si}$ nanocrystals capped with $\mathrm{SiO}_{2}$ or embedded in glass matrices fall within the shaded region in Fig. 18, albeit this region embodies a wide band of energies or wavelengths at each value of $d$. An issue for these samples ${ }^{2,3,7,3,35 a}$ is the role of the $\mathrm{SiO}_{2}$ or glass and suboxide layer that almost certainly exists at the interface. There are undoubtedly defects and surface states at this interface, and they could play a significant role in determining the luminescence from such samples. ${ }^{33}$ The various authors of these works imply generally that the observed PL is intrinsic to the Si nanocrystal cores, but doubts remain. The shaded region also embodies much of the published PL peak energies on porous Si samples, an 
observation that has been used by some authors to suggest that the PL of porous Si is intrinsic to Si nanostructures such as nano-size wires and crystallites. Strong doubts and many puzzles remain in this area as well. ${ }^{2 a, 35 b}$

Reviewing Fig. 18, we note that Risbud et al's $^{7}$ two data points (the sample associated with the lower point being larger than that for the upper by an unknown amount) lie above the shaded region. In this case, the Si nanocrystals, which had sharp, well-defined fringe images, are embedded in a complex ( $\mathrm{Si}, \mathrm{K}, \mathrm{Ba}, \mathrm{B}, \mathrm{Ca}$ ) glass matrix, not $\mathrm{SiO}_{2}$. The difference between these results and those in the shaded region may be due to differences in the two glasses, but how, or why, is not known.

Kanemitsu's ${ }^{8}$ two data points at $d=3.7 \mathrm{~nm}$ in Fig. 18 are also from surface oxidized Si nanocrystals. Under $\mathrm{cw}$ illumination he observed a red PL band centered at $750 \mathrm{~nm}(1.65 \mathrm{eV})$, which falls in the shaded region, but under laser-pulse illumination he observed a fast-decaying blue-green band centered at $\sim 500 \mathrm{~nm}(2.5 \mathrm{eV})$ in addition to the slow-decaying red band. The blue-green band energy falls way above the lower shaded region. Time-resolved PL measurements indicated that carriers generated in the Si core state are rapidly localized into the lower-energy surface states. Spectroscopic analysis suggested that the red band is caused by a hopping-limited recombination process in the surface-localized state of the nanocrystals, while the blue-green band is caused by band-edge emission from the core state of the nanocrystals. These observations and conclusions are relevant to our own results, as we shall discuss below.

Iwasaki et al' $s^{6}$ datum point at $d=4.8 \mathrm{~nm}$ in Fig. 18 is on a Si nano-colloid sample in 2propanol. In terms of the synthetic approach, this sample is the closest to ours among the results in the figure. In this case blue-green emission centered at $2.5 \mathrm{eV}$ is observed which is close to what Kanemitsu ${ }^{8}$ observed on his smaller $(d=3.7 \mathrm{~nm})$ surface-oxidized nanocrystals. However, unlike Kanemitsu, these authors attribute this PL to recombination of excitons trapped near the surface interfacial region, and not due to emission from the Si core.

Figure 18 shows some recent results from $\mathrm{Kim}^{35 \mathrm{a}}$ The samples were Si-rich silicon oxide layers produced by electron cyclotron resonance chemical vapor deposition and rapid thermal annealing. The layers contained a distribution of Si nanocrystals with sizes ranging from 1 to $3.5 \mathrm{~nm}$ with peaks in the distribution at $\sim 1,2.5$ and $3 \mathrm{~nm}$. At $300 \mathrm{~K}$, stable, reproducible PL peaks were observed at $3.4,3.08,2.62$ and $2.22 \mathrm{eV}$. The first three of these peaks exhibited the 
same temperature dependence as the Si bandgap and were attributed to recombination in the confined Si nanocrystals. The peak energies are plotted arbitrarily in Fig. 18 at $d=2.5 \mathrm{~nm}$ which corresponds to the sharpest peak in Kim's size distribution. These peak energies are seen to be very comparable to some of the PL energies for our samples.

Turning now to our data in Fig. 18 we note that whereas some of our data points fall in the shaded region, the rest of the data fall well above this region. As noted earlier in this section, our PL spectra generally exhibited a higher energy major peak and a lower energy minor peak. It is the energies of these minor peaks that fall in the shaded region. (We should mention that the observed relatively low intensities of these minor PL peaks are to a large measure due to the fact that our samples were quite dilute, $\sim 10^{-4}$ molar). The heavy dashed curve in the figure provides a good representation for the intermediate part of our data, and the data of Kanemitsu ${ }^{8}$ and Iwasaki et al. ${ }^{6}$ Our highest energy points in the figure are, we believe, associated with direct $\left(\Gamma_{25}-\Gamma_{15}\right)$ recombination. As far as we know, this is the first observation of such direct recombination from $\mathrm{Si}$ and is most likely made possible by zone-folding conduction band states from other parts of the Brillouin zone to the $\Gamma$ point on reducing the cluster size. Consistent with this conclusion, the decay lifetime of the luminescence is on the order of a nanosecond (as discussed below). Kim's highest energy point in Fig. 18 may also be due to this direct recombination.

As for our intermediate and low emission energies in Fig. 18, two scenarios can be examined. The first presumes that the low energy emissions (and thereby all emissions in the lower shaded region) are due to $\mathrm{Si}$ bandgap emission, i.e., indirect recombination. The intermediate energy emissions would then be associated with some chromophore that may be related to the synthesis. The measured PL decay lifetimes for these emissions are on the order of a few to tens of nanoseconds, as we shall see later. Alternatively, the intermediate energy emissions are due to bandgap emissions and the low energy emissions due to surface-state recombination as also suggested by Kanemitsu. ${ }^{8}$ The electron-hole pairs are generated in the interior of the Si nanocrystals. Some of the electrons rapidly decay into the lower-energy surface states while some (a larger fraction in our case) recombine with the holes across the gap One difficulty with this scenario is that some of the emission energies for sizes $d>4 \mathrm{~nm}$ fall above effective mass (EMA) predictions (discussed below). This scenario is thus difficult to accept 
given that EMA generally overestimates the quantum confinement effect--an observation that argues in favor of the first scenario.

Some observations on porous $\mathrm{Si}$ are relevant to the intermediate energy regime in Fig. 18. Blue light emission is commonly observed from aged and or oxidized porous $\mathrm{Si}$. This emission is observed in the range $410-490 \mathrm{~nm}(-3.0-2.5 \mathrm{eV})$ depending on the aging/oxidation conditions, and the radiative lifetime is in the ns range for some samples and in the $\mu$ s range for other samples. The energy range of this PL overlaps most of the data in the intermediate regime in Fig. 18. The origin of this PL is a subject of continuing debate between two competing mechanisms. In one, as in the recent work of $\mathrm{Li}$ et al, ${ }^{35 c}$ the PL with $\mu$ s lifetime is believed to come from the crystalline cores of oxidized nanometer-size Si particles, whereas the PL with ns lifetime is attributed to the oxidized layer. In the second mechanism, the PL is attributed to e-h recombination at centers located at the interface between the Si nanocrystals and the Si oxide as well as on the inside of the oxide layers. ${ }^{35 \mathrm{~d}}$ In this mechanism, the photoexcitation most likely occurs in the crystalline Si cores and the carriers transfer to the luminescent centers at the interface or in the oxide. This second mechanism comes closer to our present case where we believe that the PL in the intermediate energy regime in Fig. 18 is due to surface state recombination.

\section{Radiative Recombination Rate}

The radiative e-h recombination rate $(1 / \tau)$, where $\tau$ is the radiative lifetime, is an important measure of the effectiveness of a luminescing material. For direct bandgap semiconductors like GaAs the recombination is fast, $\tau$ being on the order of 1-10 nanoseconds or less. For indirect gap materials like $\mathrm{Si}$, on the other hand, the recombination is slow, $\tau$ being on the order of tens of microseconds to milliseconds. In nanocrystals all vibronic as well as recombination rates are expected to increase as the electron and hole wavefunctions become more compact and more overlapping with decreasing size.

We have obtained $\tau$ for a couple of our Si nanocrystal samples from PL decay measurements at $295 \mathrm{~K}$. The first experiment was performed on $d=3 \mathrm{~nm}$ nanocrystals in solvent using an apparatus with picosecond resolution in the laboratory of our collaborator Prof. David 
Kelley of Colorado State University. The results expressed as PL amplitude vs. time at $460 \mathrm{~nm}$ for excitation at $300 \mathrm{~nm}$ are shown in Fig. 19. The decay is multi-exponential but can be satisfactorily fitted by two lifetimes, a fast initial component with $\tau=48$ ps and a slow component with $\tau=1.6 \mathrm{~ns}$. It is not clear what the origin of the fast component is, but the slower is comparable to that for direct-like e-h recombination. This $\tau$ is about equal to that of direct excitonic recombination in bulk GaAs.

Figure 20 shows the PL decay kinetics for another sample with $d=3 \mathrm{~nm}$. In this case kinetics were measured on an instrument with much lower time resolution than for the sample in Fig. 21. The inset in Fig. 20 shows the temporal profile of the PL measured at $440 \mathrm{~nm}$, and the figure shows that the decay can be adequately fit by two exponentials. The fast component with $\tau=17 \mathrm{~ns}$ is convolved by the instrument response so that the decay of the sample may be actually faster. The slow component with $\tau=48 \mathrm{~ns}$ is due to the sample. The scatter at long times is due to digitization noise. As discussed above, the origin of this PL is not known, but we believe it is due to surface state recombination. This $\tau=48 \mathrm{~ns}$ is also much shorter than $\tau$ 's observed for $\mathrm{SiO}_{2}$-capped $\mathrm{Si}$ nanoclusters. For e.g., Littau et $\mathrm{al}^{2}$ fit their time-resolved PL results on their colloid 1.0 sample $(d<5 \mathrm{~nm})$ with two exponentials yielding $\tau_{1}=17 \mu$ s and $\tau_{2}=$ $76 \mu$ s. (A note of caution is in order: in Figs. 20 the decay is measured over a very narrow range of times and the $\tau$ 's should be considered tentative.)

Because of the large excitonic binding energy for Si nanocrystals, the photoluminescence is expected to be excitonic in origin rather than due to recombination of free electrons and holes. The multiexponential (or non-exponential) nature of the PL decay and the wide range of lifetimes (nanoseconds to milliseconds) observed experimentally for $\mathrm{Si}$ nanoclusters and porous Si suggest the presence of effects such as inhomogeneous size distributions, non-intrinsic radiative recombination, as well as non-radiative relaxation channels. 


\section{Photoluminescence Quantum Efficiency}

To estimate the quantum efficiency (Q.E.) of various solutions of Si nanoclusters, we measured the total area under the PL curve and normalized this by the absorbance of the sample at the excitation wavelength. We performed identical measurements on a laser dye, Coumarin 500 , known to be close to $100 \%$ efficient at light emission. We took the ratio of the dye emission PL area to the cluster solution PL area under identical excitation conditions, lamp energy, and spectrometer bandpass as a measure of the efficiency of light emission of the nanoclusters. The largest room temperature Q.E. achieved was $3.9 \%$ for $2.0 \mathrm{~nm}$ Si nanocrystals in acetonitrile with no special treatment of the cluster surface, or annealing.

In a similar study on Ge nanocrystals ${ }^{19}$ we examined the influence of the polarity of the solvent on the Q.E. Extensive data on $d=2 \mathrm{~nm}$ Ge nanocrystals revealed a gradual decrase in PL efficiency with decreasing solvent polarity (ethylene glycol $>$ acetonitrile $>$ toluene $>$ orthoxylene). The same trend is expected for $\mathrm{Si}$.

\section{Comparison With Theory}

There has been an evolution of theoretical treatments of the electronic structure of semiconducting nanocrystals. The earliest treatment is the effective mass approximation (EMA) due to Efros and Efros ${ }^{36}$ which assumed spherical nanocrystals, parabolic energy bands and infinite potential barriers at the crystal boundary. In the EMA one replaces the microscopic quasi-periodic potential of the bulk material by a constant potential, and the kinetic energy operator is replaced by an effective-mass operator derived from the parabolic expansion of the bulk band structure. Experience on a large number of materials has shown that EMA generally treats large $(d>5-6 \mathrm{~nm})$ nanocrystals fairly accurately but fails seriously for smaller nanocrystals. ${ }^{37}$ Consequently, there have been a number of attempts at improving it. For example, Brus ${ }^{38}$ included the electron-hole $(e-h)$ interaction energy, i.e., the Coulomb term, dealt with finite-potential walls to calculate excited state energies, and considered the influence of the dielectric constant of the surrounding medium. Kayanuma ${ }^{39}$ went on to consider the influence of nanocrystal shape, specifically, spheres vs. cylinders.

Despite these improvements, EMA results generally overestimate the confinement effect especially for small clusters of $\mathrm{Si}$ (as shown below) and other semiconductors. 
In an attempt to overcome some of the short comings of EMA, several authors ${ }^{40-42}$ have employed semi-empirical tight binding models. These models include the non-parabolicity of the energy bands away from zone center and generally yield better agreement between calculated band energies vs. size and experiment.

Wang and Zunger ${ }^{43}$ extended pseudopotential calculations to large ( 1000 atom) $\mathrm{Si}$ nanocrystals and included the effects of band mixing and the influence of shape and crystallographic orientation as well as the role of the surface in determining quantum confinement. Among their key findings are the following: (1) there is no appreciable influence of shape on the bandgap vs. size for quantum spheres, cubes and rectangular boxes; (2) the band edge states, i.e., the highest occupied molecular orbitals and the lowest unoccupied molecular orbitals of Si nanocrystals are bulk-like, not surface-like, consistent with our experimental results; (3) the bandgap is insensitive to the surface orientation of the nanocrystal; and (4) unlike the bandgap, the radiative lifetime is sensitive to the shape and orientation of the nanocrystal.

Empirical pseudopotential methods have also been employed to treat $\mathrm{Si}$ nanocrystals. Rama Krishna and Friesner ${ }^{18}$ calculated the influence of size confinement on the indirect gap (excitonic transition) as well as on the $\Gamma_{25}-\Gamma_{15}$ and $\Gamma_{25}-L$ transitions. An interesting aspect of their work is the finding that the direct $\Gamma_{25}-\Gamma_{15}$ transition energy first increases slightly with decreasing crystal size and then decreases. The decrease is attributed to the inverted parabolic shape of the conduction band at $\Gamma$. As noted earlier, our results do not agree with this finding. Rather, we find a significant increase in this energy for our smallest nanocrystals.

Another feature of Rama Krishna and Friesner's results is the finding that quantum size confinement splits the two overlapping $\left(\mathrm{p}_{\sigma^{*}}\right)$ conduction bands at $\Gamma\left(\Gamma_{15}\right)$ as well as the two overlapping bands at the top of the valence band also at $\Gamma$. (Actually, these two latter bands are slightly split in bulk Si by spin-orbit interaction as shown in Fig. 8). These splittings should, in principle, be reflected in that part of the absorption spectrum due to direct transitions at $\Gamma$. However, as we have already noted, there is no discernible evidence for it in our Si nanocrystal spectra. The reason for the absence of such evidence is embodied in the results in Fig. 8, namely the curvature of the two split-off conduction bands at $\Gamma$. Specifically, the first split-off band is 
concave and the second is convex providing a potential well for electrons. Thus, the first direct optical transition at $\Gamma$ in Si nanocrystals is to this second band.

Figure 21 shows various theoretical results on the variation of the indirect bandgap energy with size for Si nanocrystals. Clearly, there are very substantial quantitative differences among these results, especially at small sizes. In attempting to compare these results with experimental data, we note that very few authors have reported the experimental magnitude of the bandgap for Si clusters. Almost all of the available data consist of PL peak energies vs. size as was presented in Fig. 18. Thus, we superimpose on the theoretical results in Fig 21 the experimental data of Fig. 18 in an attempt to look for trends. We caution, however, that many of the experimental data shown are not nesessarily attributed to recombination associated with the (indirect) bandgap, rather, they may be due to surface or impurity recombination or, in the case of some of our results, to direct recombination at $\Gamma$. Additionally, in comparing the experimental and theoretical results, one needs to keep in mind the relatively large and size-dependent exciton binding energy as discussed above.

Unfortunately, the differences among various model predictions as well as the large differences among the experimental PL peak energies in Fig. 21 makes it difficult to draw any meaningful conclusions. As noted earlier, a very serious deficiency in the experimental data is the large uncertainty in determining size.

Zunger and $\mathrm{Wang}^{28}$ compared their calculated exciton energy (including Coulomb interaction between the excited electron and hole) vs. size both with bandgaps estimated from absorption measurements and with experimental PL peak energies and found some systematic trends. (Our results are, of course, not included in this comparison). Specifically, all the experimental bandgap data fell above the calculated Energy vs. $d$ curve whereas all the PL data fell below this curve. There are serious uncertainties in this comparison, as the authors acknowledge. These include the large uncertainty in determining $d$ experimentally, the usually wide distribution of sizes in samples and the difficulty of determining the indirect gap from experimental data on clusters (as discussed earlier). Despite these difficulties the comparison is systematic, and the trend revealed is interesting. These observations led the authors to conjecture that the observed PL originates from some persistent (approximately size-independent) defects or impurity states (e.g., surface states) rather than from intrinsic nanocrystal states. This is said to 
be supported by the fact that the experimental gap deduced from the absorption spectrum is systematically larger than the experimental PL energy, as we have found in the present work. However, we should caution that in Zunger and Wang's comparison the absorption and PL data came from different sources, and, furthermore, the absorption data are from porous Si samples, making definitive conclusions difficult (especially in view of the above-mentioned experimental uncertainties).

Although many of our findings appear to be in qualitative agreement with Zunger and Wang's observations, we feel that it is premature to come to any definitive conclusions about mechanisms. The experimental situation, especially with respect to size determination and the ability to obtain monodisperse nanocrystals with controlled surfaces, has to greatly improve before we reach that stage.

Leung and Whalley $^{29}$ investigated $e-h$ interactions in truncated small spherical $\mathrm{Si}$ nanocrystals by incorporating Coulomb, exchange and spin-orbit couplings into tight-binding models. They reported the optical absorption spectra for nanocrystals with 41,83 , and 147 atoms in size, corresponding to $d=1.16,1.47$ and $1.78 \mathrm{~nm}$, respectively, the latter being equal in size to our smallest nanocrystals. The nanocrystals were constructed by sequentially adding shells about a central atom, and therefore have tetrahedral symmetry. Figure 22 compares their calculated $\epsilon_{2}$ vs. energy spectrum for the 147 -atom cluster with the extinction data for our $1.8 \mathrm{~nm}$ nanocrystals which are of comparable size. ( $\epsilon_{2}$ is the imaginary part of the dielectric constant). The absorption spectrum, $\sigma$ abs $(\omega)$, is directly related to $\epsilon_{2}(\omega)$ by

$$
\sigma_{\mathrm{abs}} \sim \omega \epsilon_{2}(\omega)
$$

The agreement between the two spectra in Fig. 22 is fairly good, especially with respect to the bandgap and the first direct $\left(\Gamma_{25}-\Gamma_{15}\right)$ transition.

Ögüt et $\mathrm{al}^{32}$ calculated the optical gap of Si nanocrystals as a function of size from $\sim 3 \mathrm{~nm}$ to $1 \mathrm{~nm}$ from first principles using a real-space pseudopotential method. The calculations were performed on hydrogen-passivated spherical Si clusters. The results yield larger quantum confinement shifts in the gap than other model calculations, as shown in Fig. 21, as well as large excitonic Coulomb energies, as noted earlier. 
Theoretically, the intrinsic recombination rate is given by ${ }^{42}$

$$
\frac{1}{\tau}=\frac{4}{3} \frac{\alpha \omega n}{m_{c}^{2} c^{2}}\langle i|p| f\rangle
$$

where $n$ is the refractive index, $\omega$ is the photon angular frequency, $\alpha=e^{2} / \hbar c$, and the matrix element $\langle i|p| f\rangle$ connects the purely electronic wave functions between the initial and final states. Several groups have calculated $\tau$ as a function of size using different models for bandgap recombination in Si nanocrystals. Some of these results are summarized in Fig. 23 which also shows our experimental results as well as those of other authors.

Because of afore-mentioned uncertainties in the experimental data, particularly in size determination, it had been hoped that model calculations will provide guidance as to the dependence of $\tau$ on size. Unfortunately, the very large disparities among the various model results in Fig. 23 do not allow us to draw any definitive conclusion. The only certainty in these results is that $\tau$ decreases with decreasing cluster size, as is expected. Leung and Whaley ${ }^{29}$ provided two bounds for $\tau$ vs. $d$ as shown in the figure. The bounds account for variations in model parameters for both surface-truncated and hydrogen-terminated nanocrystals. Most all of the other calculated results fall between these two bounds.

As for the experimentally-determined $\tau$ 's, we can make the following observations. First we note that the model results in Fig. 23 are for bandgap recombination. Three data points on $\mathrm{SiO}_{2}$-capped $\mathrm{Si}$ nanocrystals (one from Wilson et $\mathrm{al}^{10}$ and two, presumably on the same sample, from Littau et $\mathrm{al}^{2}$ ) fall in the middle of the band of model results. The associated $\tau$ 's are on the order of a few tens of microseconds. We also show a band of experimental results ${ }^{1}$ on porous $\mathrm{Si}$. These data also fall within the broad band of model results. Our two data points ( $\tau$ 's $=1.5$ and $50 \mathrm{~ns}$ ) and Kanemitsu's datum point ( $\tau=0.8 \mathrm{~ns}$ ) fall well outside the range of the model results as they should, consistent with our interpretation of the associated PL as being due to direct e-h recombination and surface/defect recombination and not due to bandgap recombination. There are no model calculations appropriate for these data. 


\section{SUMMARY AND CONCLUSIONS}

We have successfully grown size-selected Si nanocrystals in the size range 1.8 to $10 \mathrm{~nm}$. Controlled nucleation and growth was achieved by dissolving anhydrous ionic halide salts ( $\mathrm{SiX}_{4}$, where $\mathrm{X}=\mathrm{Cl}, \mathrm{Br}$ or I) in the hydrophilic interior of inverse micellar cages and reducing the $\mathrm{Si}(\mathrm{IV})$ ions to $\mathrm{Si}(0)$ using strong reducing agents such as anhydrous metal hydrides (e.g. $\mathrm{LiAlH}_{4}$ ).

High resolution TEM fringe images show that the nanocrystals are of high crystalline quality, and electron diffraction results show that they retain their bulk diamond structure down to about 4-5 nm diameter. Optical absorption data suggest that these nanocrystals retain their bulk-like properties and structure down to the smallest sizes produced $(\sim 1.8 \mathrm{~nm}$ diameter containing about $150 \mathrm{Si}$ atoms).

High pressure liquid chromatography (HPLC) techniques with on-line optical and electrical diagnostics were developed to purify and separate the clusters into chemcially pure, monodisperse populations. These techniques proved to be crucial to obtaining background-free absorbance and PL spectra which allowed assignments of the observed optical transitions based on band theory results and estimates of the energy shifts associated with quantum confinement effects. In addition to the long wavelength absorption tail associated with the indirect-bandgap, several direct transitions were evident in the data. It was found that the smallest gap in nanosize Si remains indirect to the smallest sizes studied, $d=1.8 \mathrm{~nm}$.

The different electronic transitions exhibited various quantum confinement effects. The indirect bandgap shifts from $1.1 \mathrm{eV}$ in the bulk to $\sim 2.1 \mathrm{eV}$ for nanocrystals $\sim 2 \mathrm{~nm}$ in diameter, and the direct transition at $\Gamma\left(\Gamma_{25}-\Gamma_{15}\right)$ blue shifts by $0.4 \mathrm{eV}$ from its $3.4 \mathrm{eV}$ bulk value over the same size range. Some transitions were relatively insensitive to cluster size, a feature that can be qualitatively understood in terms of the shape of the associated dispersion curves in the band structure.

The blue shift of the indirect bandgap with decreasing cluster size is in qualitative agreement with certain theoretical predictions. However, the wide variations in various theoretical models of nanosize Si coupled with considerable uncertainty in size determination for clusters less than 3-4 nm, made it impossible to choose among competing models. 
We have observed room temperature photoluminescence from Si nanocrystals at various wavelengths in the range $700-350 \mathrm{~nm}(1.8-3.5 \mathrm{eV})$, i.e. across the visible range. The largest quantum efficiencies (compared to coumarin dye molecules) were $\sim 4 \%$ for $d=2 \mathrm{~nm} \mathrm{Si}$. No post synthesis surface treatment was employed to achieve these results.

Solvent polarity was shown to influence quantum efficiency. ${ }^{19}$ Measurements (largely on $\mathrm{Ge}$ and some on Si nanocrystals) in ethylene glycol, acetonitrile, toluene and ortho-xylene show a gradual, but small decrease in PL efficiency with decreasing solvent polarity, while the PL mean energy and shape was invariant.

The most intense PL was in the blue region of the spectrum $(-400 \mathrm{~nm})$ and is attributed it to direct electron-hole recombination at $\Gamma$ ( $\Gamma_{25}-\Gamma_{15}$ transition). The short radiative lifetime for this PL ( $t=1.5 \mathrm{~ns}$ with a faster initial component) is comparable to that for GaAs and is consistent with our assignment. This is the first observation of such direct recombination in $\mathrm{Si}$ and is a consequence of quantum confinement.

A relatively strong PL centered around $580 \mathrm{~nm}$ is fairly insensitive to cluster size, and is attributed to surface (or defect) recombination. Other PL peaks were tentatively assigned (including a weak PL attributed to indirect recombination at the bandgap). More work is needed to confirm these assignments.

The work presented here represents our first exploratory attempts to understand the relationship between Si nanocluster size, structure, surface chemistry and the resulting optical properties. We have identified features in the optical absorption and PL of nanosize Si that deserve much more detailed study in order to understand the effect of size and surface termination on the optical and electronic properties. Especially with the smallest sized clusters studied, many of the concepts of, for example, band structure, derived from assumptions of translational symmetry of the lattice may seem inappropriate, and the nature of the bonding at the cluster surface should play a significant role in the optical and electronic properties. However, it is surprising how well the optical features of Si nanoclusters as small as $2 \mathrm{~nm}$ can be identified with bulk optical properties. Since experimental methods of studying the surface characteristics and bonding arrangement of nanosize clusters are nearly non-existent, it may be some time before experimental observations and the predictions of modern theoretical models of quantum 
confinement can be more quantitative. We assert that HPLC, which is very sensitive to small changes in surface characteristics, may play an important role in elucidating the role of surface structure on the optical properties of nanoclusters.

\section{Acknowledgement}

This work was supported by the Division of Materials Sciences, Office of Basic Energy Sciences, U.S. Department of Energy and by a Laboraatory Directed R\&D project under Contract DE-AC04-AL8500. Sandia is a multiprogram Laboratory operated by Sandia Corporation, a Lockheed Martin Company, for the Department of Energy. 


\section{References}

1. See, e.g., S. S. Iyer and Y. H. Xie, Science 260, 40 (1993) and references therein.

2. (a) K. A. Littau, P. J. Szajowski, A. J. Muller, A. R. Kortan and L. E. Brus, J. Phys. Chem. 97, 1224 (1993);. (b) L. E. Brus et al, J. Am. Chem. Soc. 117, 2915 (1995) and references therein;

3. H. Takagi, H. Ogawa, Y. Yamazaki, A. Ishizaki and T. Nakagiri, Appl. Phys. Lett. $\underline{56}, 2379$ (1990).

4. M. Yamamoto, K. Hayashi, K. Tsunetomo, K. Khono and Y. Saka, Jap. J. Appl. Phys. 30, 136 (1991) and Y. Osaka et al, Jap. J. Appl. Phys. 1ㅡ, L565 (1992).

5. W. A. Saunders, P.C. Sercel, R.B. Lee, H. Atwater, K.J. Vahala, R.C. Flanagan and E.J. Escorsi-Aparcio, Appl. Phys. Lett. $\underline{63}, 1549$ (1993).

6. S. Iwasaki, T. Ida and K. Kimura, Jap J. Appl. Phys. 35, L551 (1996).

7. S. H. Risbud, L. C. Liu and J. F. Shackelford, Appl. Phys. Lett. 63, 1648 (1993).

8. Y. Kanemitsu, Phys. Rev. B $\underline{49}, 16845$ (1994).

9. C.W. White, S.P. Withrow, A. Meldrum, J.D. Budai, D.M. Hembree, J.G. Zhu, D.O. Henderson and S. Prawer, Proc. 1998 Spring MRS Meeting (to appear); see also J. Appl, Phys. $\underline{78}, 4386$ (1995).

10. W. L. Wilson, P. F. Szajowski and L. E. Brus, Science 262, 1242 (1993).

11. A. P. Alvisatos, MRS Bulletin, P. 23, August 1995 and references therein.

12. J. P. Wilcoxon, U.S. Patent \#5,147, 841, issued September 15, 1992.

13. J. P. Wilcoxon, R. L. Williamson and R. J. Baughman, J. Chem. Phys., $\underline{98}, 9933$ (1993). 
14. J. P. Wilcoxon and G. A. Samara, Phys. Rev. Bs1, 7299 (1995); see also J. P. Wilcoxon, P. P. Newcomer and G. A. Samara, Sol. State Commun. $\underline{98}, 581$ (1996).

15. J.P. Wilcoxon and S.A. Craft, in NanoStructured Materials, Vol. 9, 85-88, Elsevier Science Ltd., (1997).

16. See, e.g., S.M. Sze, Physics of Semiconductor Devices. Wiley-Inferscience (New York, 1969) Chapt. 2 and references therein.

17. M. L. Cohen and T. K. Bergstresser, Phys. Rev. 141, 789 (1966).

18. M. V. Rama Krishna and R. A. Friesner, J. Chem. Phys. 96, 873 (1992).

19. J.P. Wilcoxon and G.A. Samara, to be published.

20. J. R. Heath, J. J. Shiang and A. P. Alivisatos, J. Chem. Phys. 101, 1607 (1994).

21. See, e.g., J. I. Pankove, Optical Processes in Semiconductors (Dover, New York, 1975), Chapt. 2.

22. M. F. Jarrold and V. A. Constant, Phys. Rev. Lett. $\underline{67}, 2994$ (1991).

23. E. Kaxiras and K. Jackson, Phys. Rev. Lett. 1, 727 (1993).

24. U. Rothlisberger, W. Andreoni and M. Parrinello, Phys. Rev. Lett. 72, 665 (1994).

25. K. D. Rinnen and M. L. Mandich, Phys. Rev. Lett. 69 , 1823 (1992).

26. N. Binggeli and J. R. Chelikowsky, Phys. Rev. Lett. 7 5, 493 (1995).

27. J. R. Haynes and W. C. Westphal, Phys. Rev. 101, 1676 (1956).

28. A. Zunger and L.W. Wang, Appl. Surf Science 102, 350 (1996).

29. K. Leung and K. B. Whaley, Phys. Rev. B $\underline{56}, 7455$ (1997).

30. T. Takagahara and K. Takeda, Phys. Rev. $3 \underline{46}, 15578$ (1992). 
31. See, A. J. Read, R.J. Needs, K.J. Nash, L.T. Canham, P.D.J. Calcott, and A. Qteish, Phys. Rev. Lett. 69, 1232 (1992); T. Ohno, K. Shiraishi and T. Ogawa, Phys. Rev. Lett. 69, 2400 (1992).

32. S. Ögüt, J. R. Chelikowsky and S. G. Louie, Phys. Rev. Lett. 79, 1770 (1997).

33. G. Allan, C. Delerue and M. Lannoo, Phys. Rev. Lett. 76, 2961 (1996).

34. S. Schuppler, S. L. Friedman, M.A. Marcus, D.L. Adler, Y.H. Xie, F.M. Ross, T.D. Harris, W.L. Brown, Y.J. Chabal, L.E. Brus and P.H. Citrin, Phys. Rev. Lett. 72, 2648 (1994).

35a. K. Kim, Phys. Rev. B 푸, 13072 (1998).

35b. Y. Kanemitsu, H. Uto and Y.Masumoto, Phys. Rev. B. 48. 2827 (1993).

35c. P. Li, G. Wang, Y. Ma and R. Fang, Phys. Rev. B 58, 4057 (1998).

35d. G. G. Qin, X. S. Liu, S. Y. Ma, J. Lin, G. Q. Yao, X. Y. Lin, and K. X. Lin, Phys. Rev. B $\underline{55} 12,876(1997)$.

36. Al. L. Efros and A. L. Efros, Sov. Phys. Semicond. 16,772 (1982).

37. See e.g., A. D. Yoffee, Adv. In Phys. 42,173 (1993) for a review.

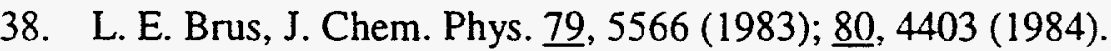

39. Y. Kayanuma, Phys. Rev. B 요, 9797 (1988); 4ㅗ, 13085 (1991).

40. Y. Wang and N. Herron, J. Phys. Chem. 91, 257 (1987); 92, 4988 (1988).

41. P. E. Lippens and M. Lannoo, Phys. Rev. B $\underline{39}$, 10935 (1989); 41 , 6079 (1990).

42. N. A. Hill and K. B. Whaley, Phys. Rev.(c ) Lett. 75 , 1130 (1995).

43. L. W. Weng and A. Zunger, J. Phys. Chem. 100, 2394, (1994) and references therein.

44. M. S. Hybertsen, Phys. Rev. Letters $\underline{72}, 15141$ (1994) and references therein. 


\section{Figure Captions}

Figure 1. Coplot of the absorbance signal (dashed line) at $250 \mathrm{~nm}$ and the refractive index signal, $\Delta \mathrm{n}$, (solid line) versus elution time using a ODS200 c18 reverse phase column and acetonitrile as the mobile phase. The Si nanoclusters were passivated with octylamine.

Figure 2. Coplot of optical absorbance at $300 \mathrm{~nm}$ (dashed curve) and conductivity (solid line) vs. elution time for a reaction mixture of $\mathrm{Si}$ nanocrystals synthesized in a cationic surfactant. Chromatography conditions were a Delta-pak-c4 (butyl-terminated reverse phase) column with $300 \AA$ pores, using tetrahydrofuran as a mobile phase at $1.0 \mathrm{ml} / \mathrm{min}$ flow. The tailing peak is typical of cationic surfactants, which tend to adhere to the column.

Figure 3. Coplot of optical absorbance at $250 \mathrm{~nm}$ (dashed line) and PL (emission detector, at $400 \mathrm{~nm}$, excitation at $250 \mathrm{~nm}$, solid line) vs. elution time for a solution containing two sizes of Si nanocrystals. Chromatography conditions were a ODS200-c18 (c18-terminated reverse phase) column with $120 \AA$ pores, using acetonitrile as a mobile phase at $0.5 \mathrm{ml} / \mathrm{min}$ flow.

Figure 4. HRTEM of a single $d=2.0 \mathrm{~nm}$ Si nanocluster showing the atomic lattice fringes.

Figure 5. HRTEM of a field of 8-10 nm Si nanoclusters.

Figure 6. SAD pattern from field of $8-10 \mathrm{~nm}$ Si nanoclusters.

Figure 7. Measured absorption coefficients near and above the absorption edge of Si. After Ref. 16.

Figure 8. The band structure of Si near its bandgap. The zero of energy is taken at the top of the valence band. After C Kittel, Introduction to Solid State Physics, John Wiley and Sons (New York 1966), Chapter 10. 
Figure 9. The extinction spectra of several Si nanocrystal samples compared with the absorption spectrum of bulk Si and a Mie theory calculation for $d=10 \mathrm{~nm} \mathrm{Si}$ taken from Ref. 10.

Figure 10. Comparison of the extinction spectra of several of our Si nanocrystal samples with results from the literature.

Figure 11. The extinction and PL (excitation at $490 \mathrm{~nm}$ ) spectra for a $d=8-10 \mathrm{~nm} \mathrm{Si}$ nanocrystal sample.

Figure 12. The absorption spectrum of a $d=2 \mathrm{~nm}$ Si nanocrystal sample. The spectrum of bulk $\mathrm{Si}$ is shown for comparison.

Figure 13. The photodissociation spectrum of Si30 clusters from Ref. 25 is coplotted with the absorption (or extinction) spectra for bulk $\mathrm{Si}$ and one of our ( $d=2 \mathrm{~nm}$ ) nanocrystal samples.

Figure 14. Plot of $(\alpha h v)^{1 / 2}$ vs. energy for $d=1.8 \mathrm{~nm}$ Si nanocrystals. The intercept of the straight line with the $\mathrm{x}$-axis defines the indirect bandgap energy.

Figure 15. The PL spectrum of an [as prepared (i.e., not chromatographed)] $d \leq 5 \mathrm{~nm} \mathrm{Si}$ nanocrystal sample. The dashed curve is for $\mathrm{d}<5 \mathrm{~nm}$ Si nanoclusters capped by $\mathrm{SiO} 2$ excited at $350 \mathrm{~nm}$, taken from Ref. 2.

Figure 16. Co-plot of the extinction and PL spectra (for two excitation wavelengths) for a $d=2 \mathrm{~nm}$ Si nanocrystal sample.

Figure 17. Coplot of the extinction and PL spectra for a $d=4.0 \mathrm{~nm}$ Si nanocrystal sample.

Figure 18. Summary of data (literature and present work) on peak PL energy versus $\mathrm{Si}$ nanocrystal size.

Figure 19. Decay of the PL intensity at $460 \mathrm{~nm}$ for a $d=3 \mathrm{~nm}$ Si nanocrystal sample. 
Figure 20. Decay of the PL intensity at $\lambda>400 \mathrm{~nm}$ for a $d=0 \times \mathrm{nm}$ Si nanocrystal sample. The inset shows the temporal profile of the PL.

Figure 21. Comparison of experimental PL peak energies and theoretical energy gap $v$ predictions for Si nanocrystals.

Figure 22. Comparison of the extinction spectrum for a $d=1.8 \mathrm{~nm}$ Si nanocrystal sample with the calculated $\varepsilon_{2}(w)$, spectrum for 147-atom Si nanocrystals (from Ref. 29). The two nanocrystal samples are of about the same size. The arrow indicates the location of the calculated indirect band gap.

Figure 23. Summary of the available (present work and literature) data on the dependence of the PL decay lifetime on Si nanocrystal size. Theoretical results are also shown. 


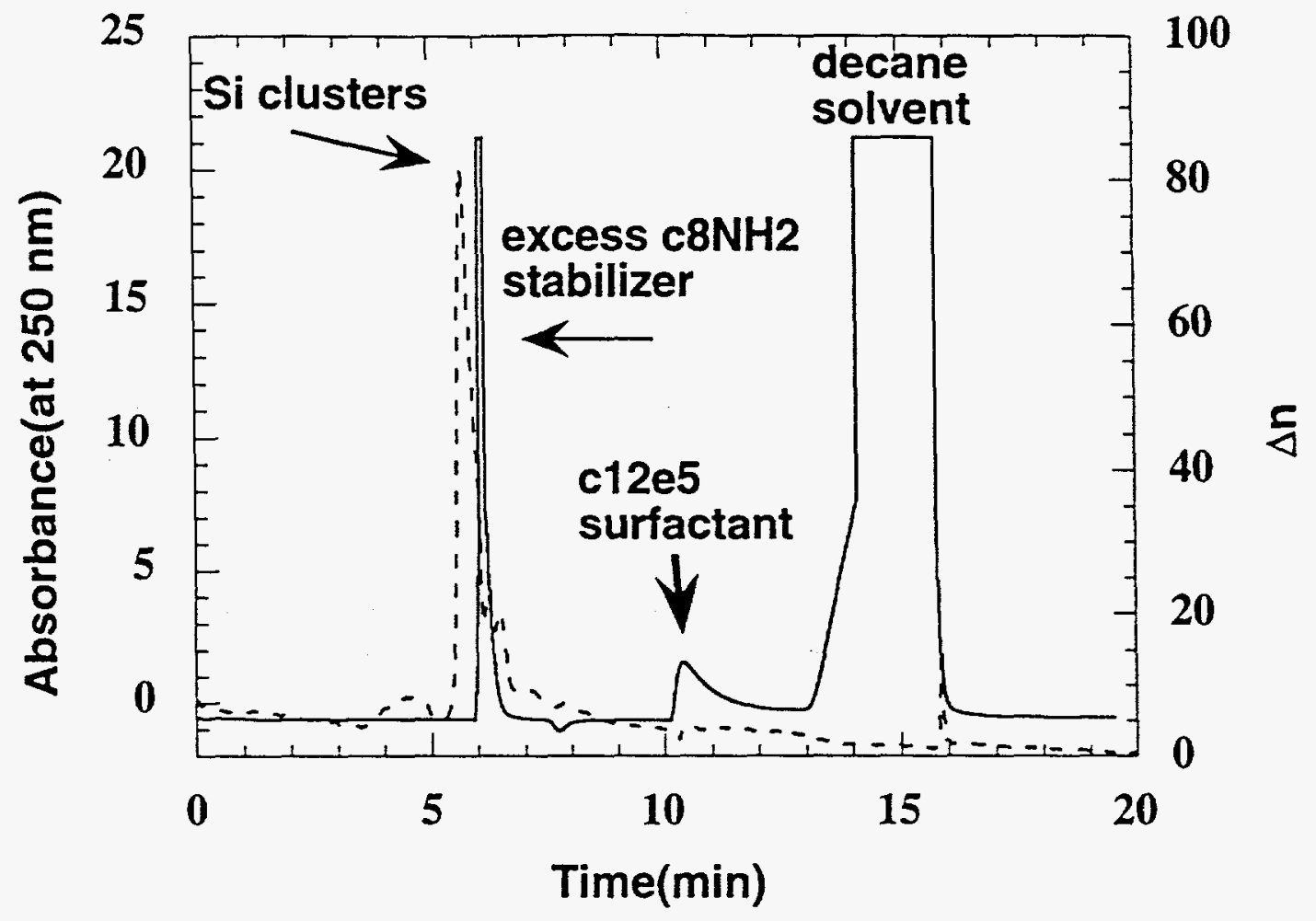

Figure 1

Wilcoxon et al

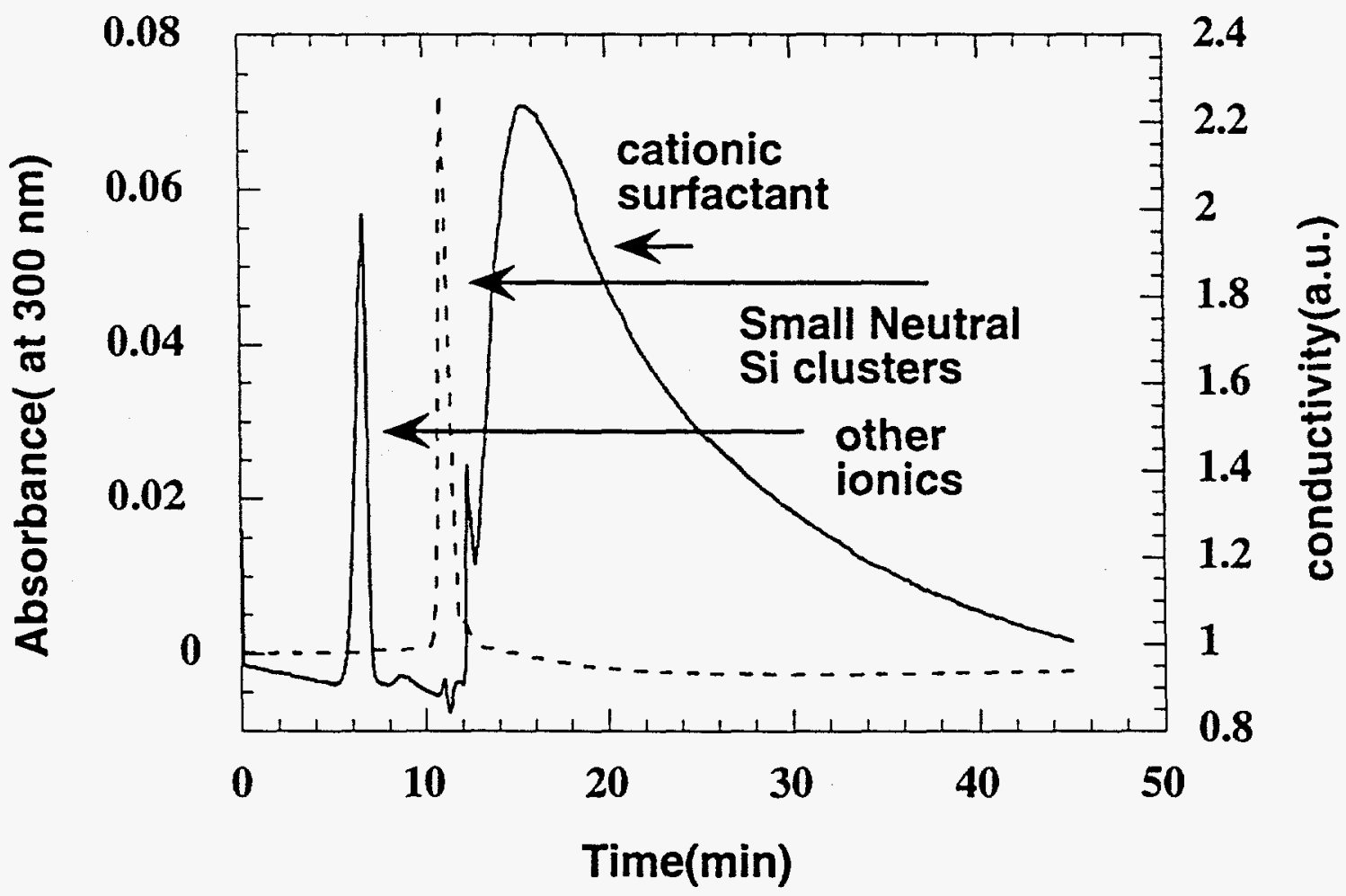

Figure 2

Wilcoxon et al 


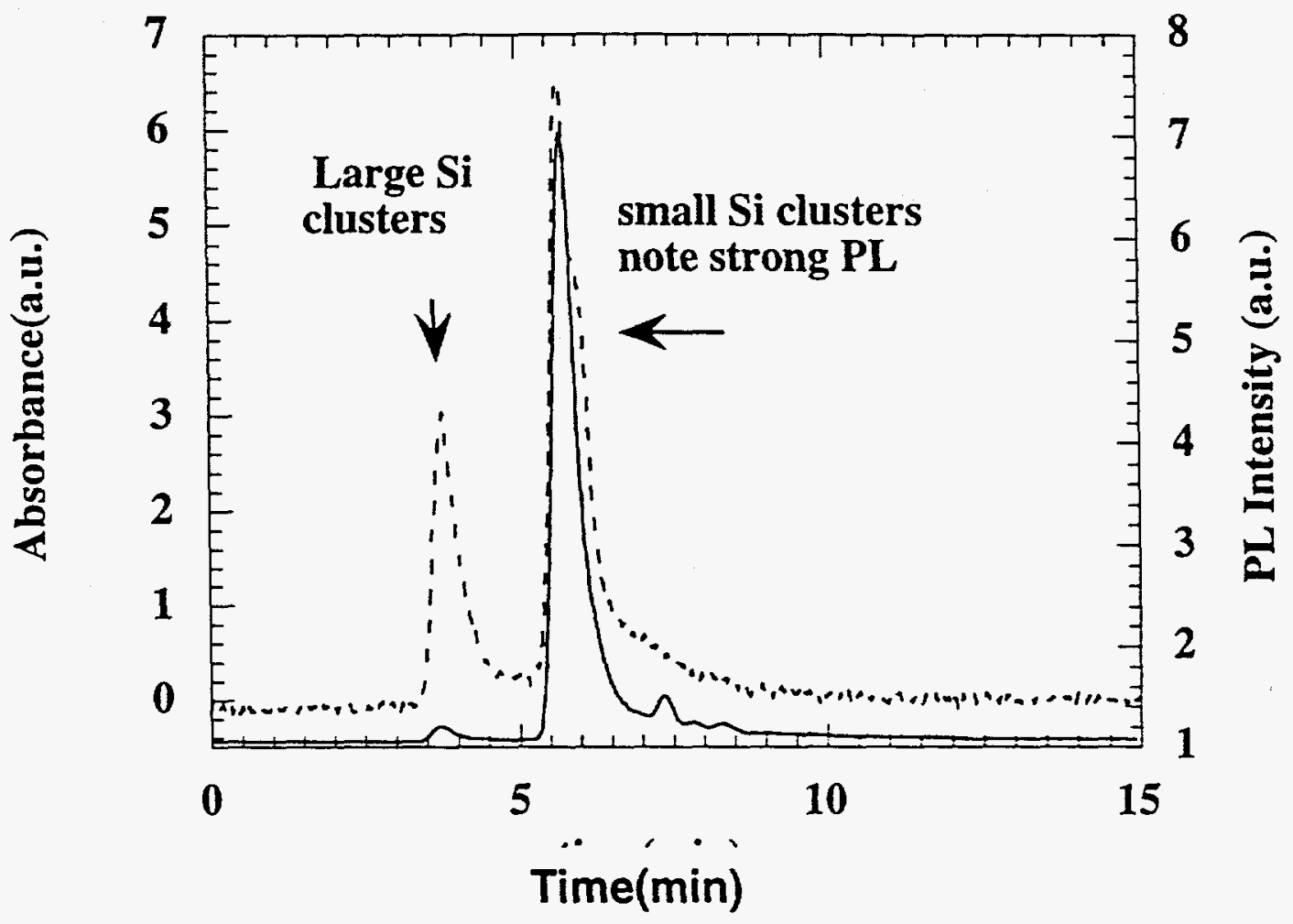

Figure 3

Wilcoxon et al

Figure 4

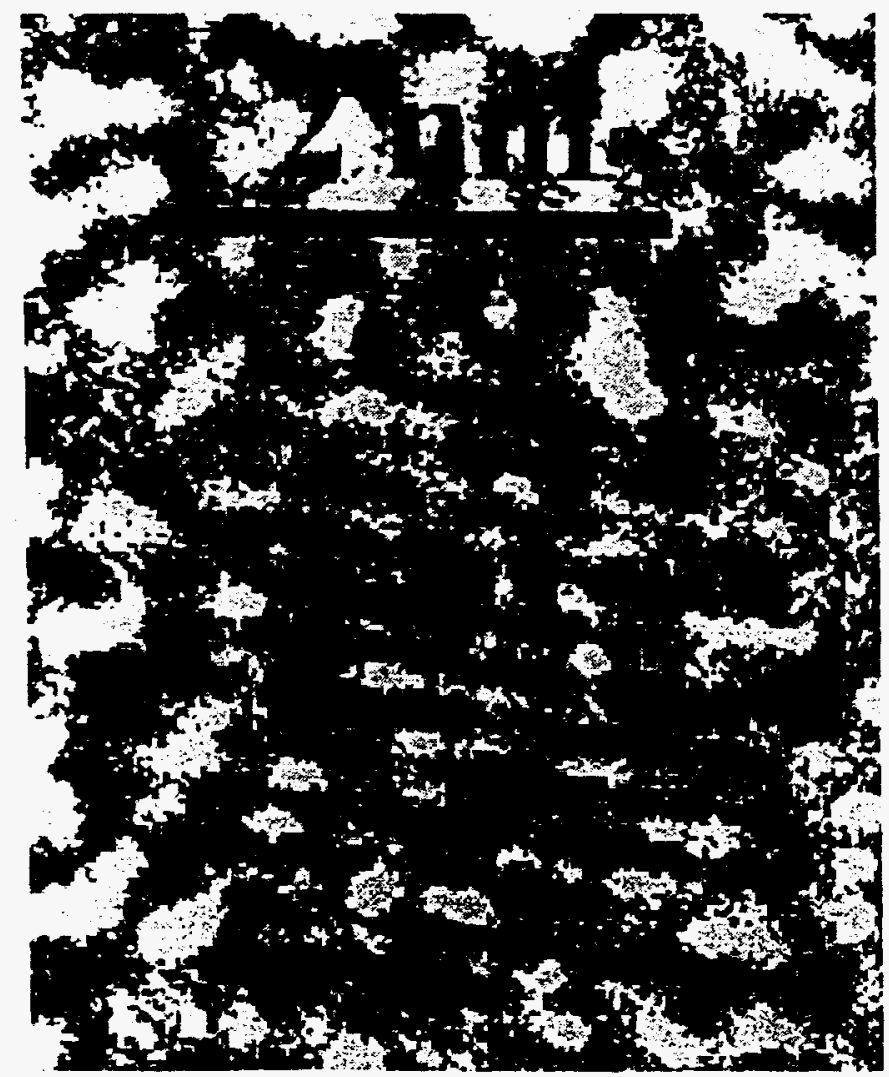

Wilcoxon et al 
Figure 5

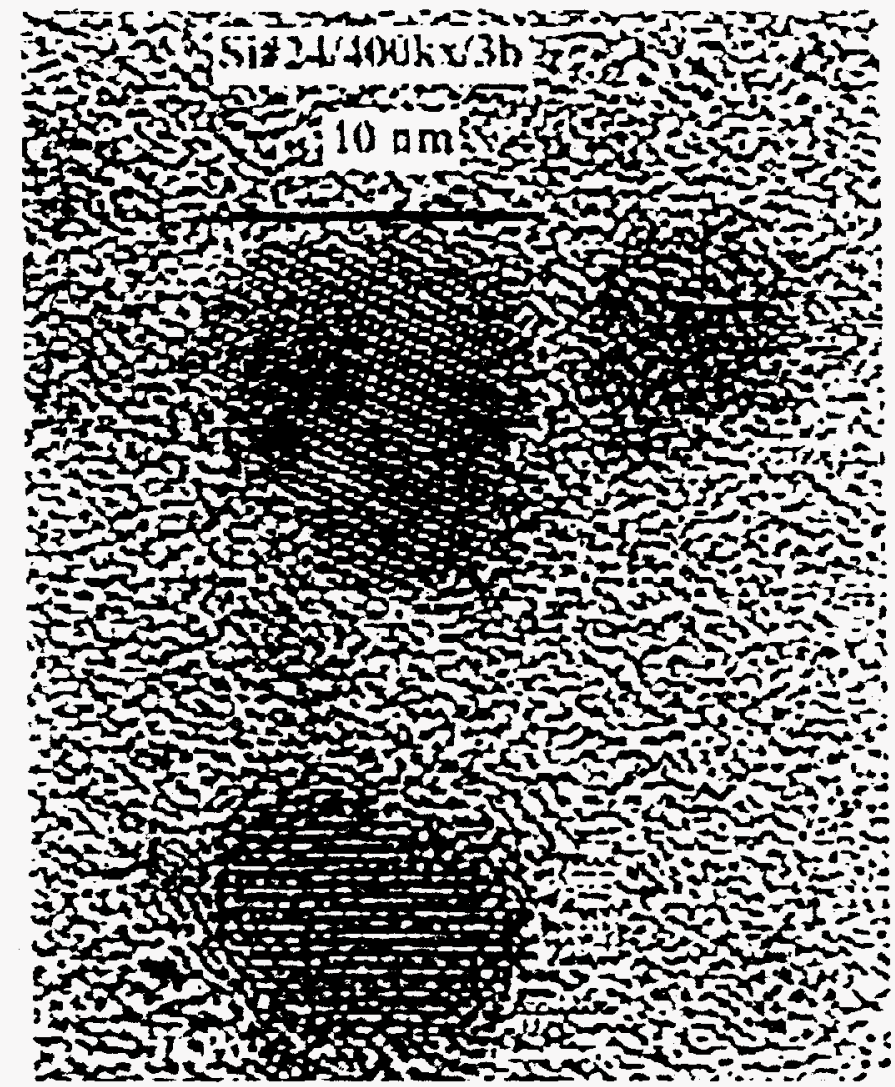

Wilcoxon et al

Figure 6

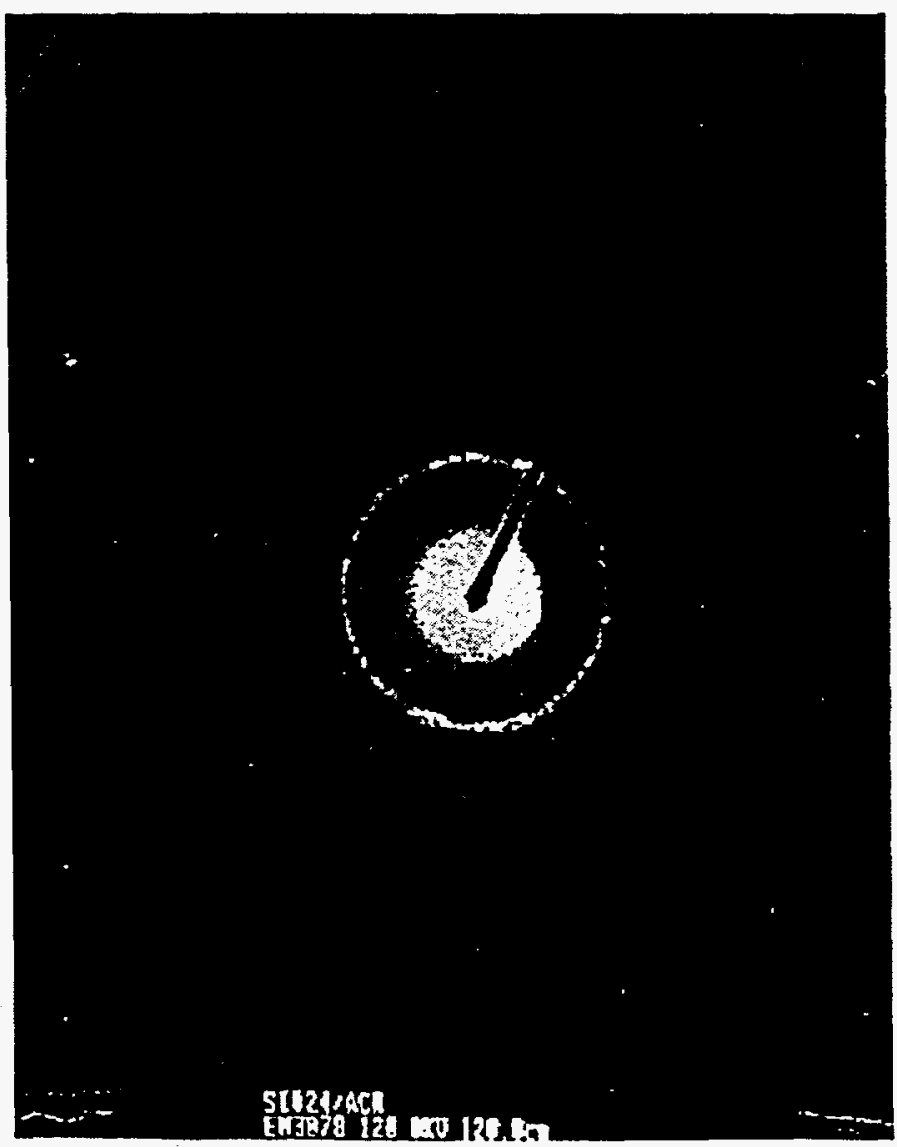

Wilcoxon et al 


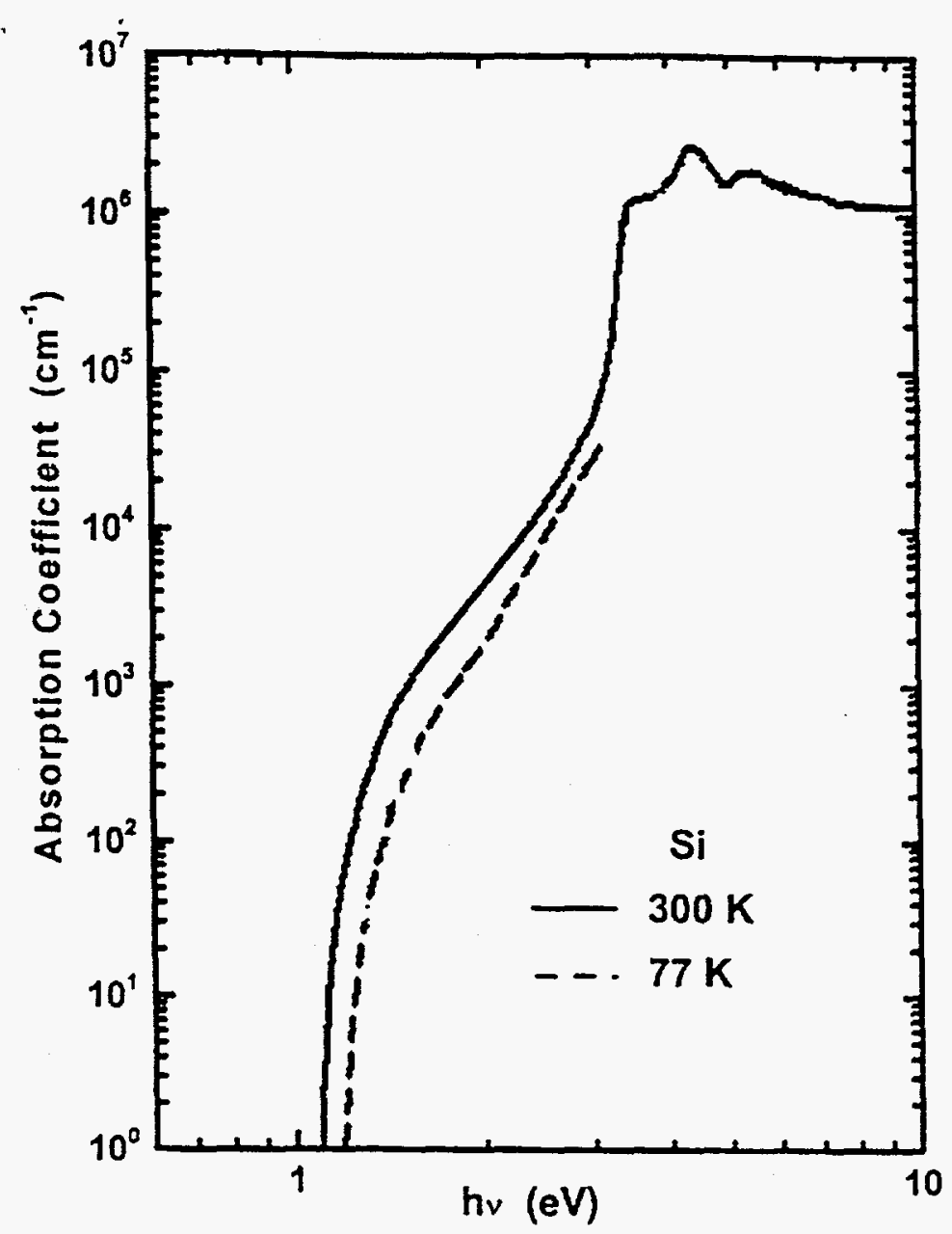

Figure 7

Wilcoxon et al

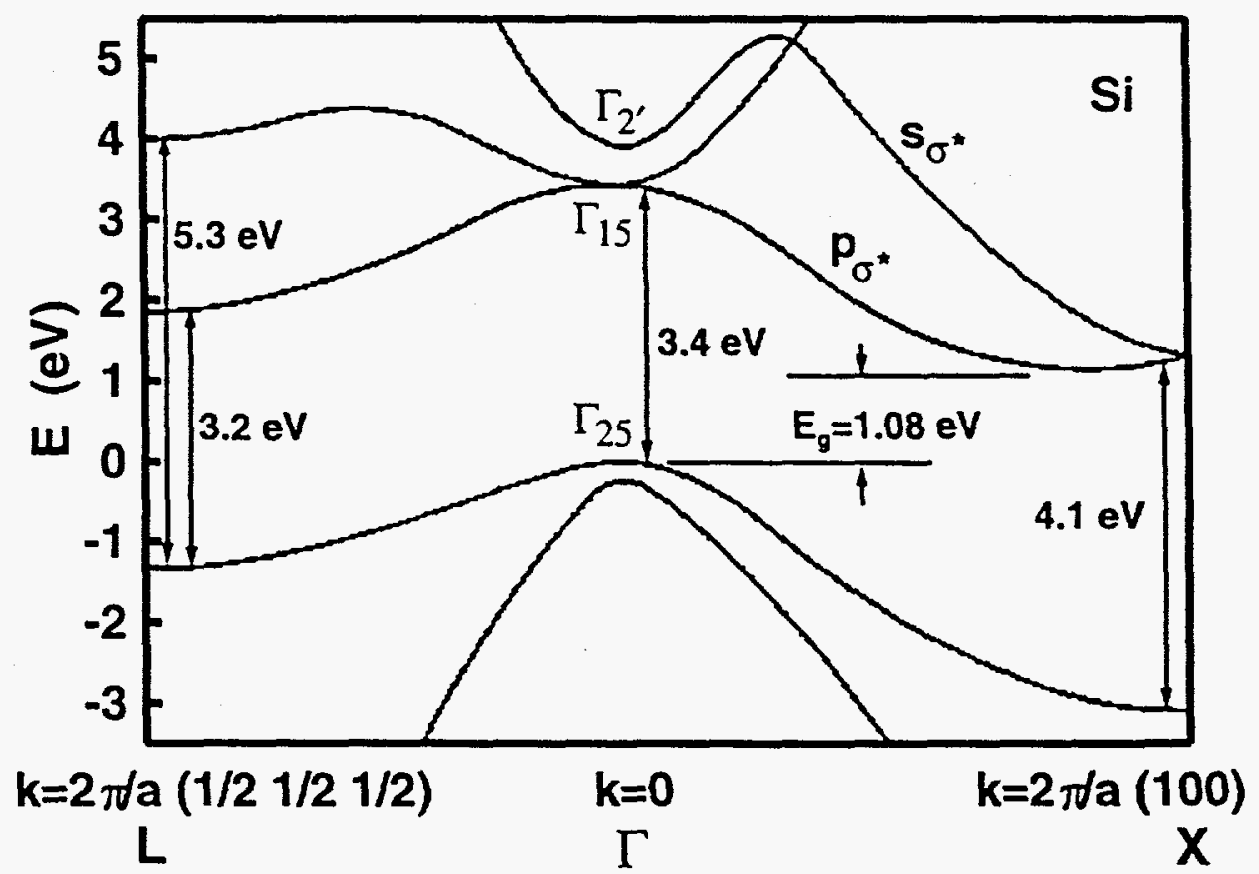

Figure 8

Wilcoxon et al 


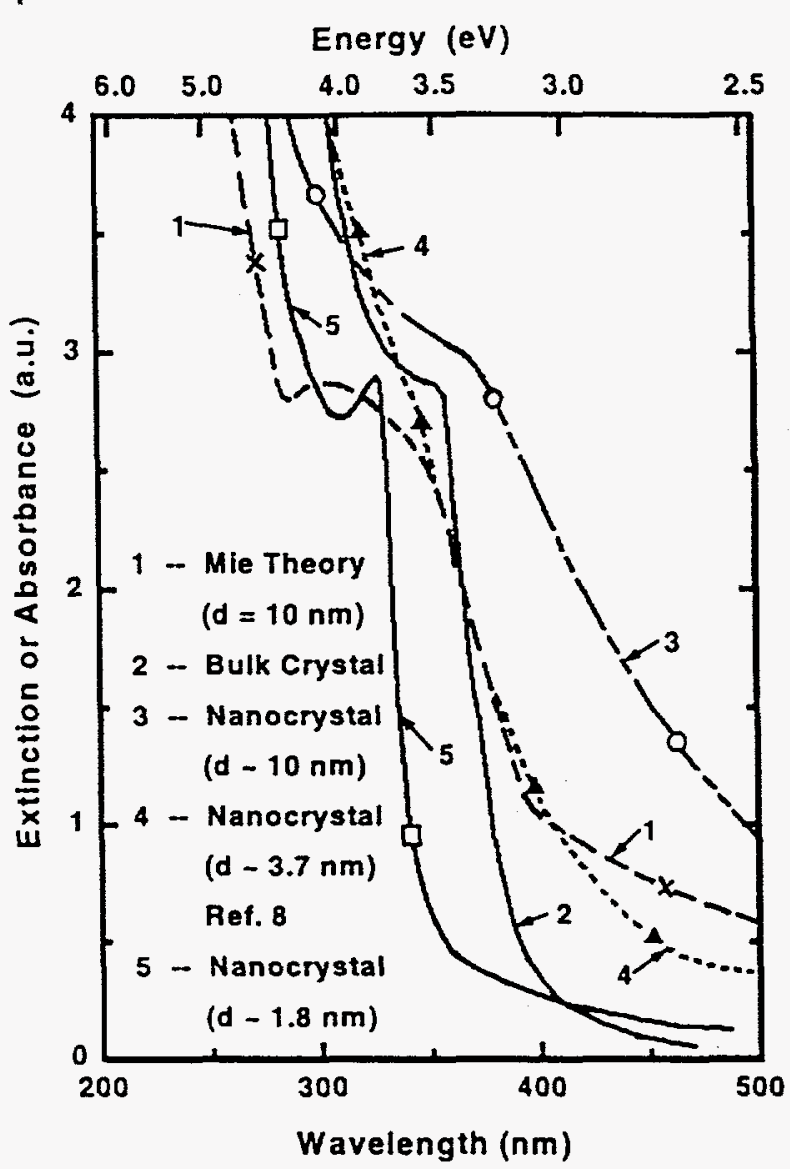

Figure 9

Wilcoxon et al

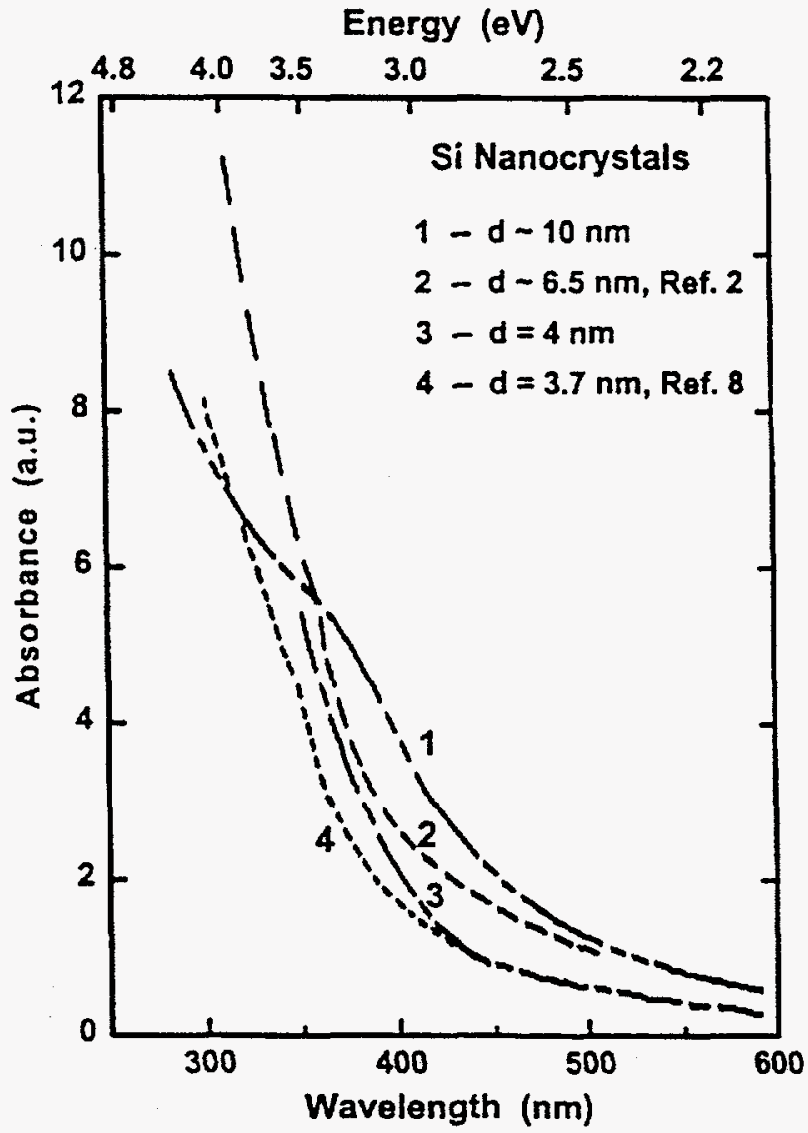

Figure 10

Wilcoxon et al 


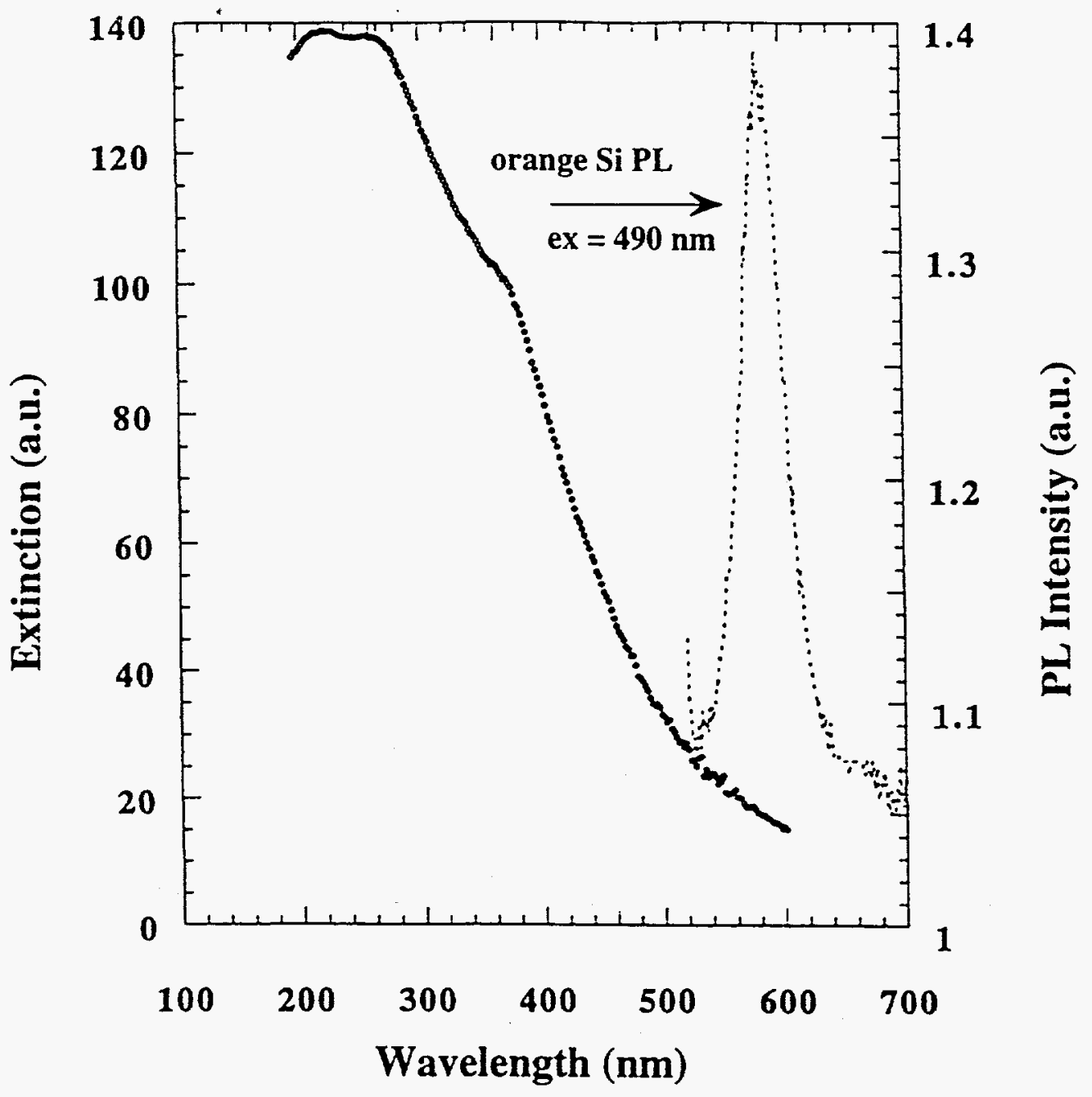

Figure 11

Wilcoxon et al

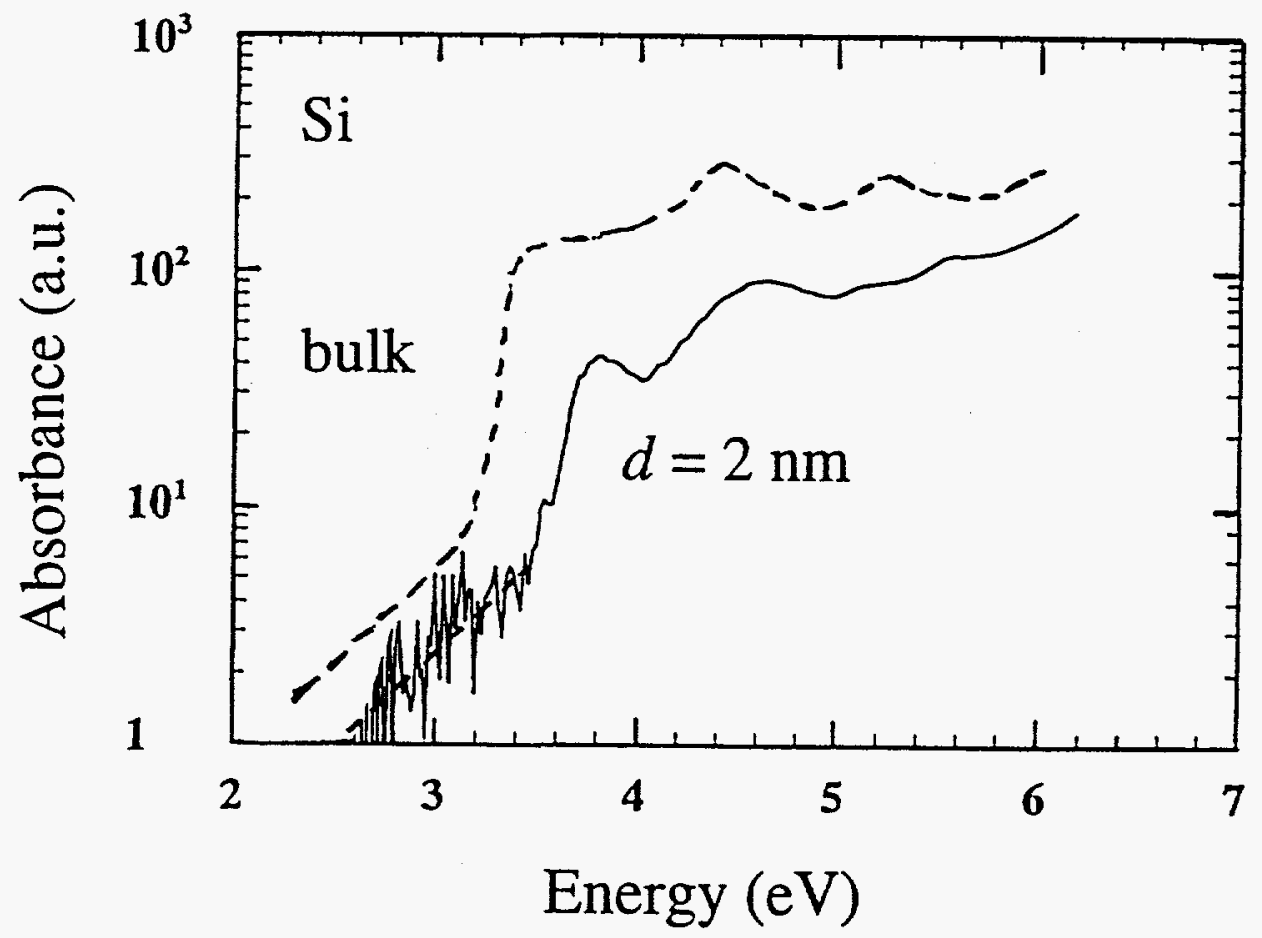

Figure 12

Wilcoxon et al 


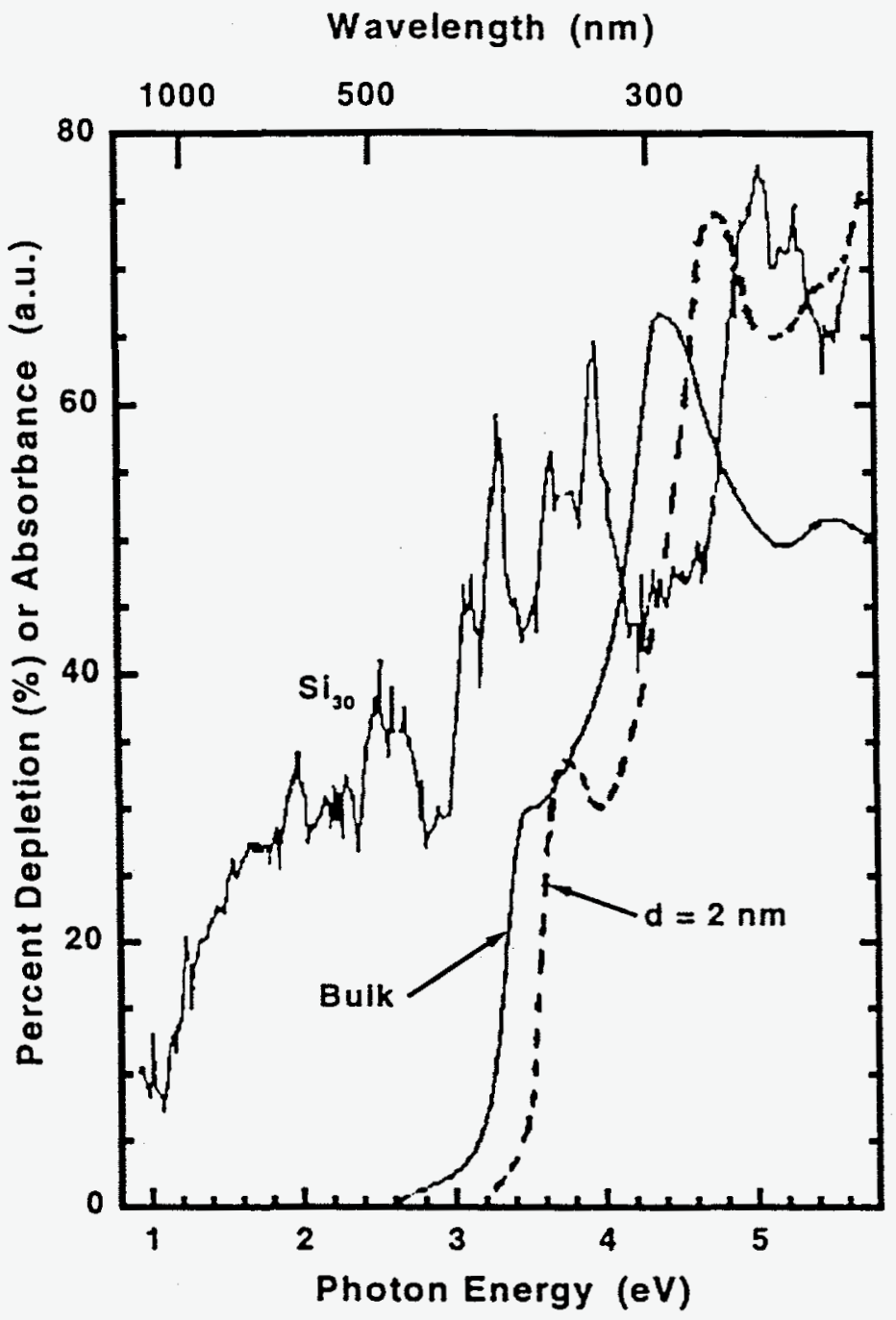

Figure 13

Wilcoxon et al

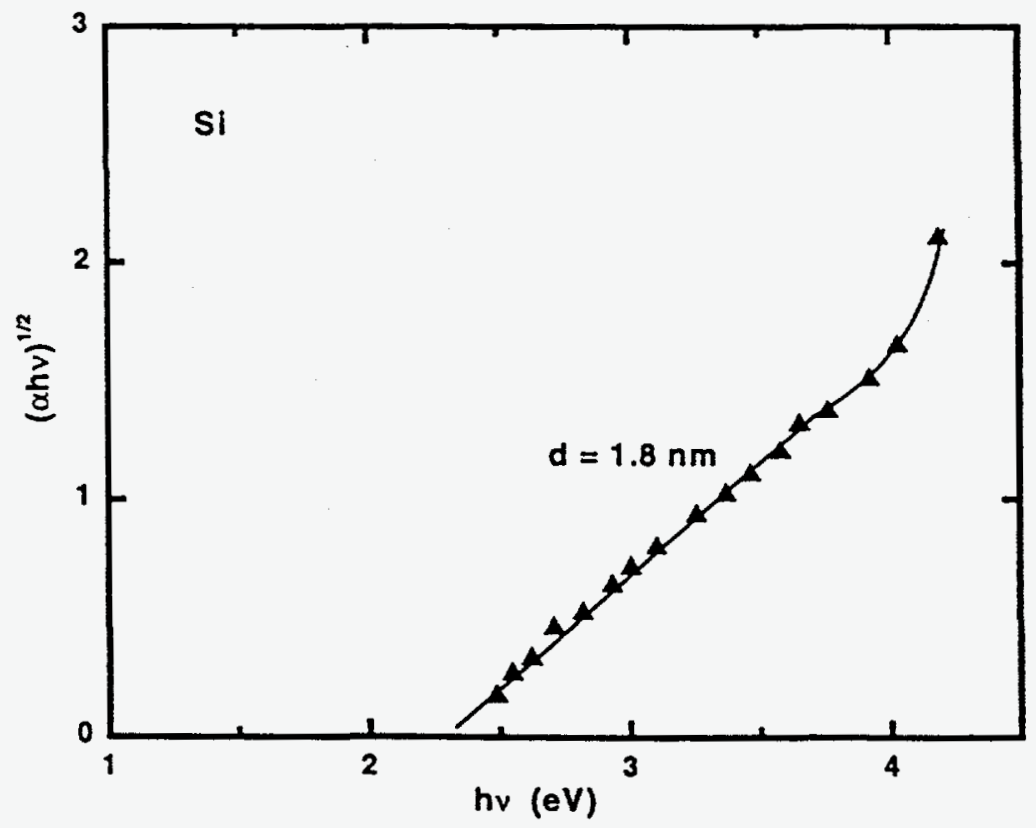

Figure 14

Wilcoxon et al 


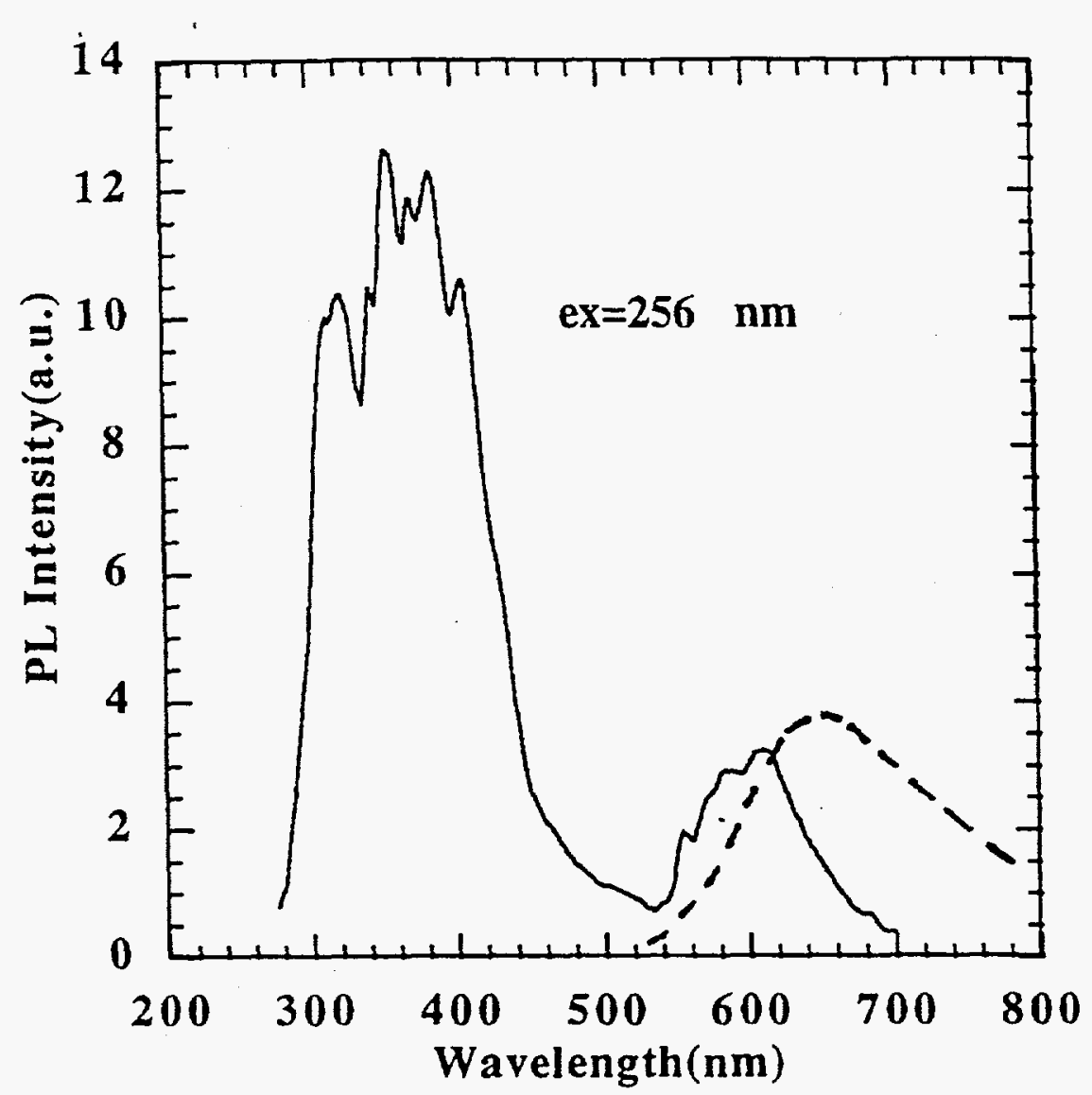

Figure 15

Wilcoxon et al

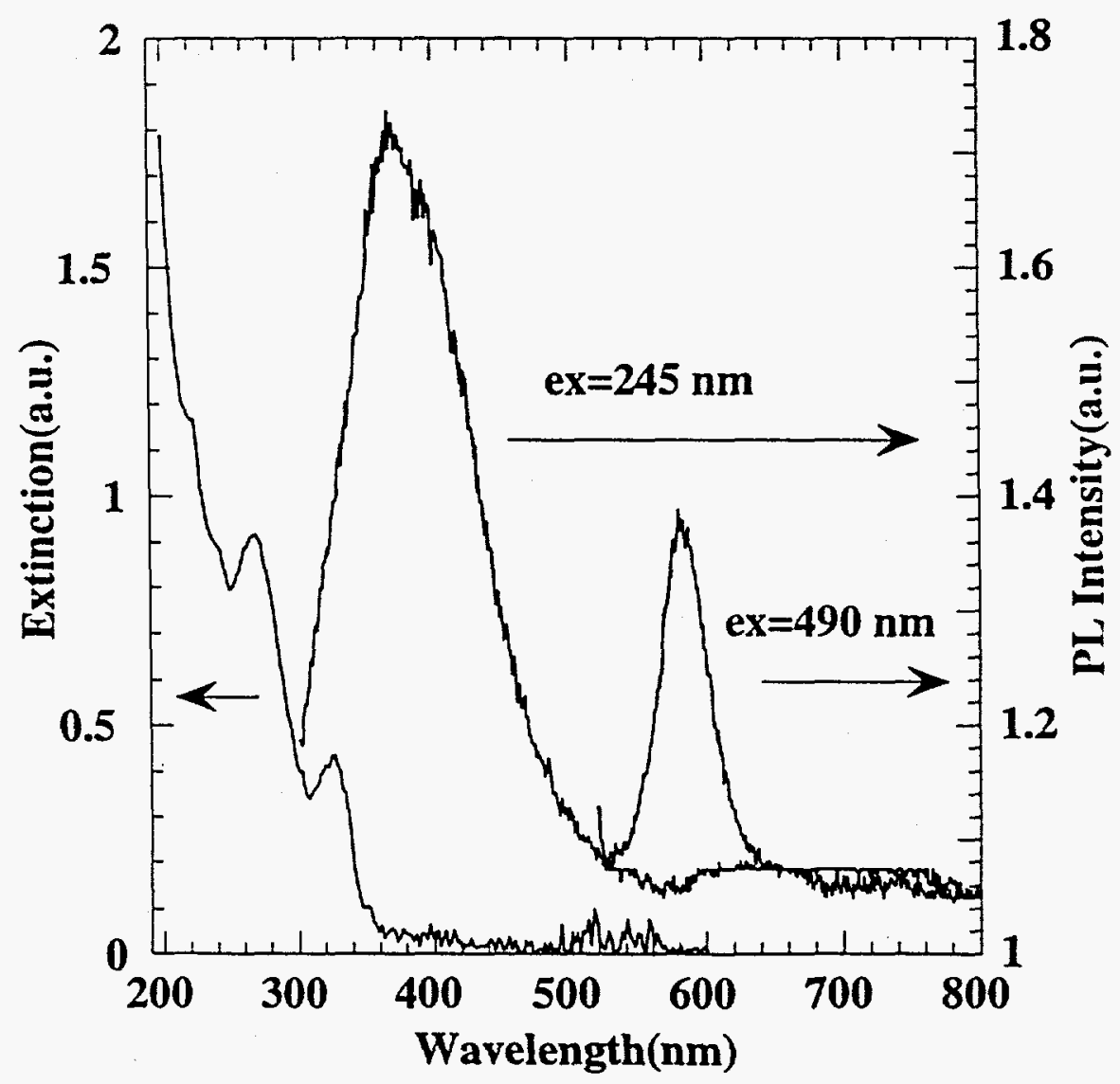

Figure 16

Wilcoxon et al 


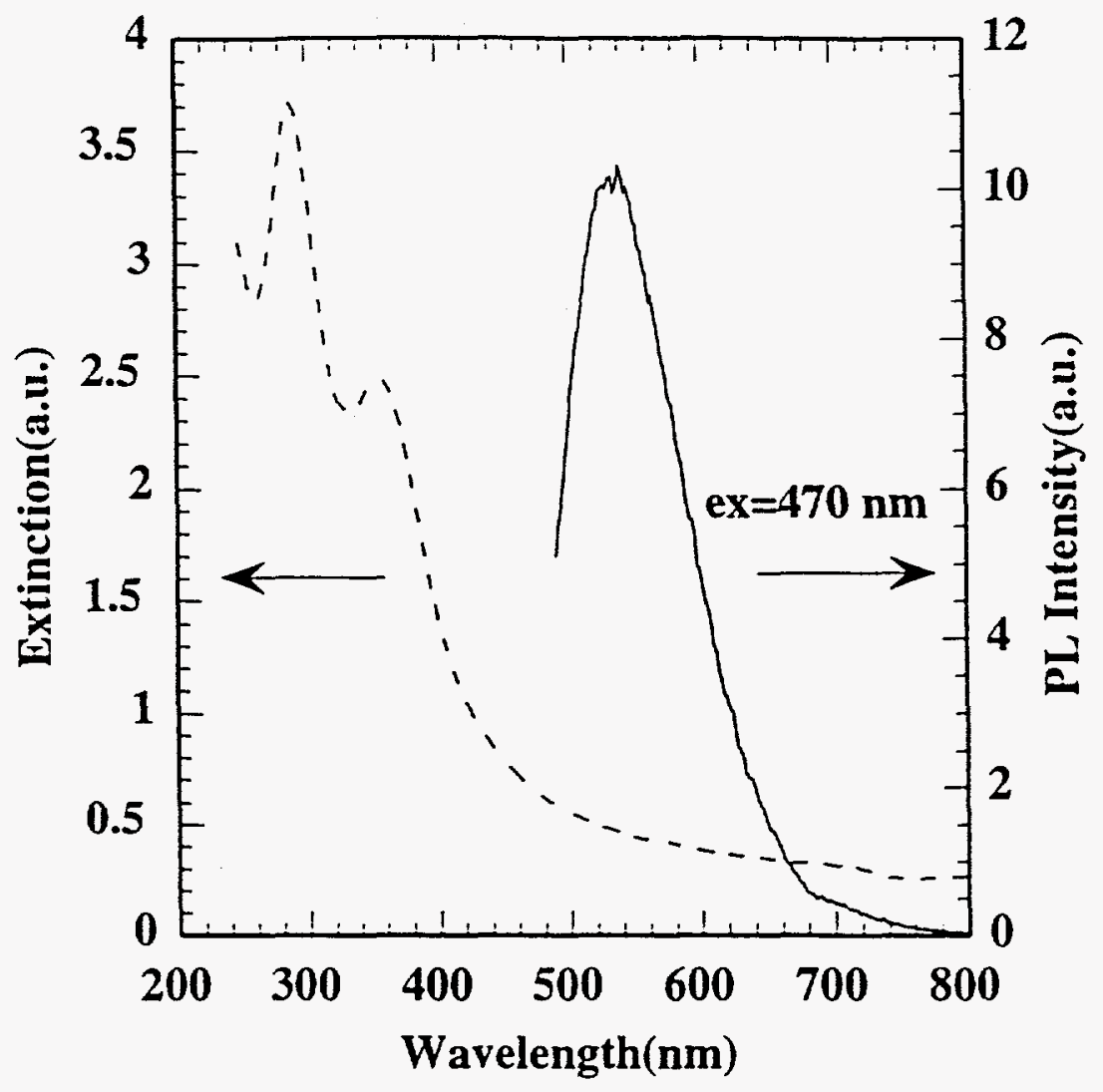

Figure 17

Wilcoxon et al

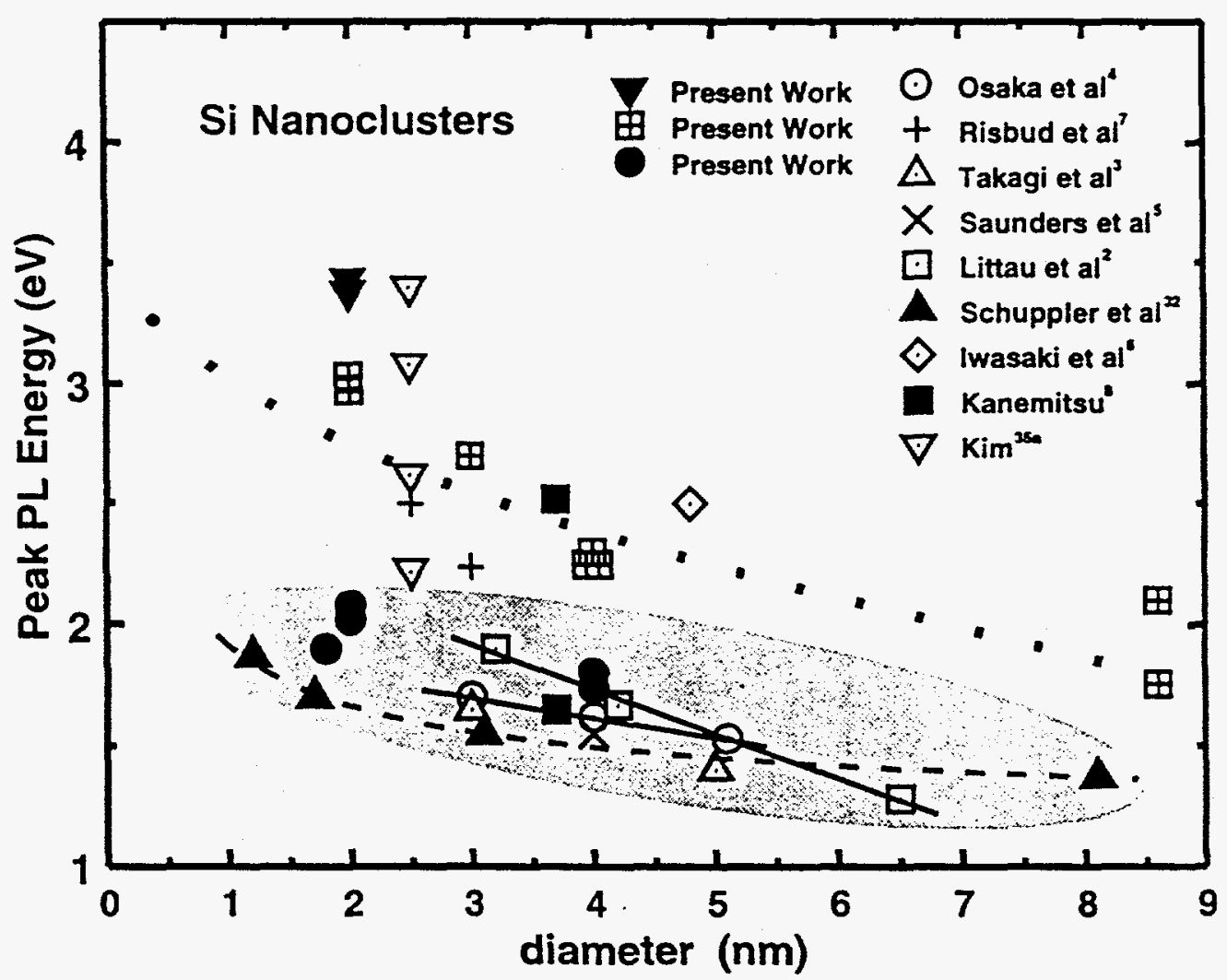

Figure 18

Wilcoxon et al 


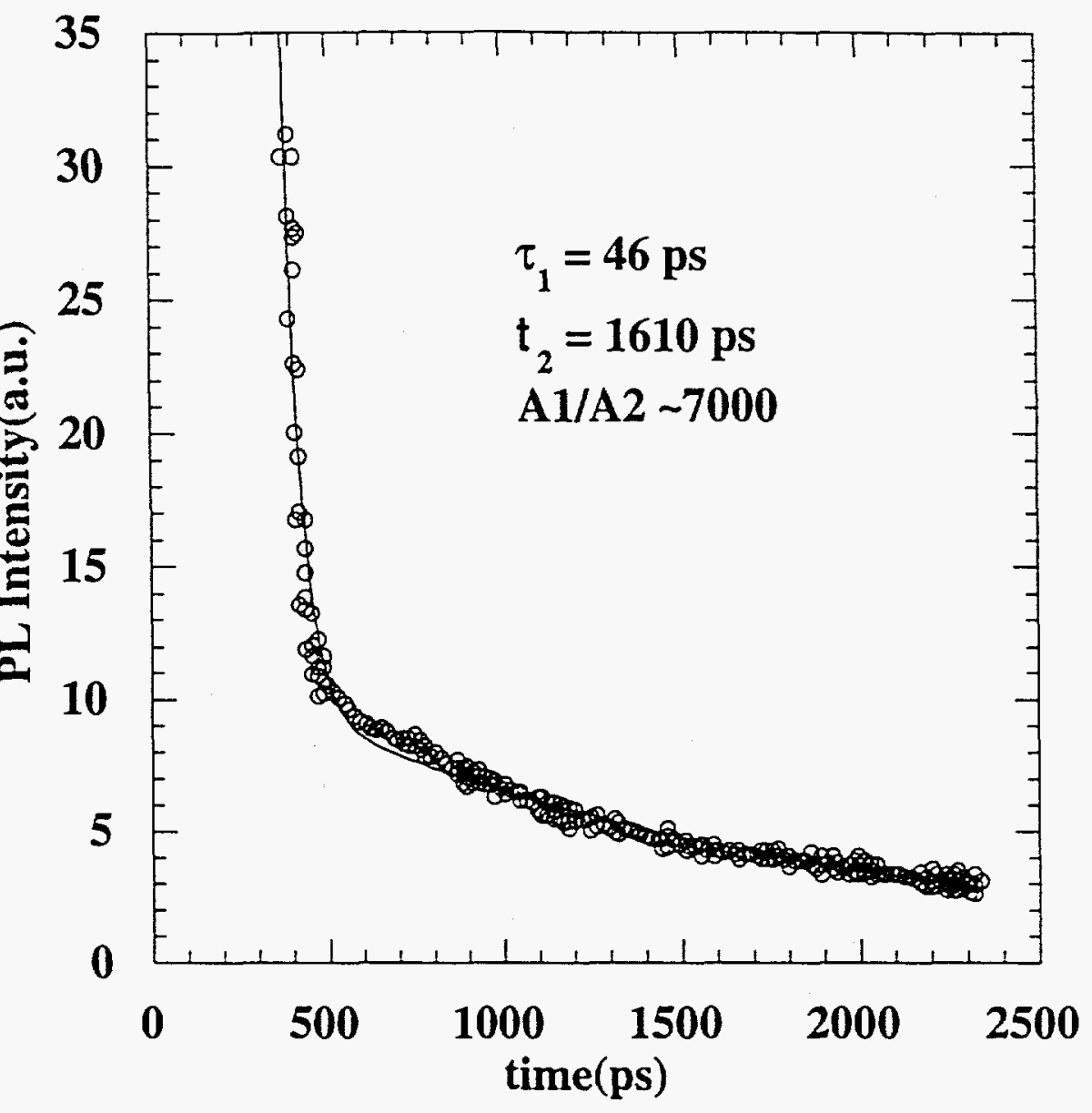

Figure 19

Wilcoxon et al

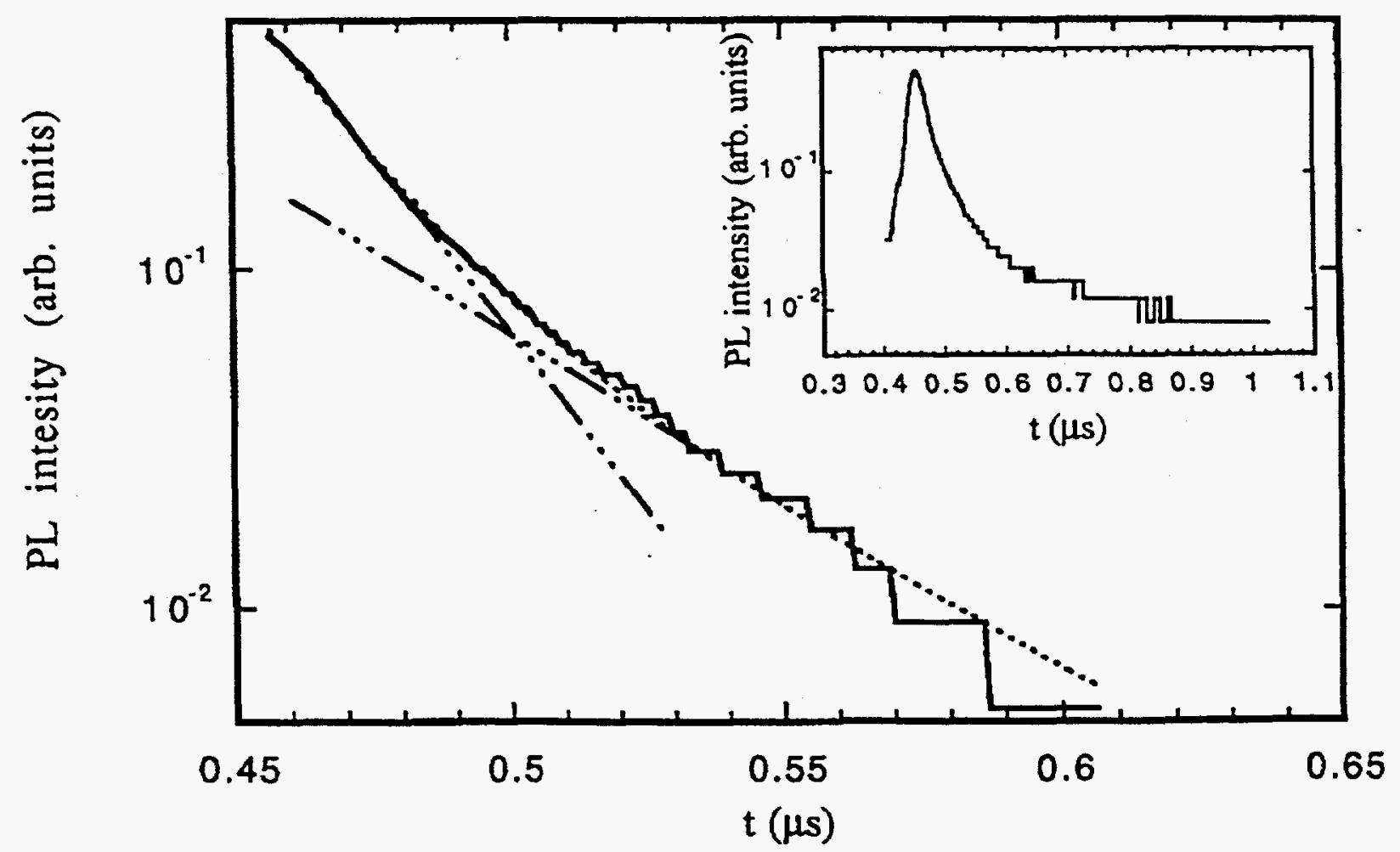

Figure 20

Wilcoxon et al 


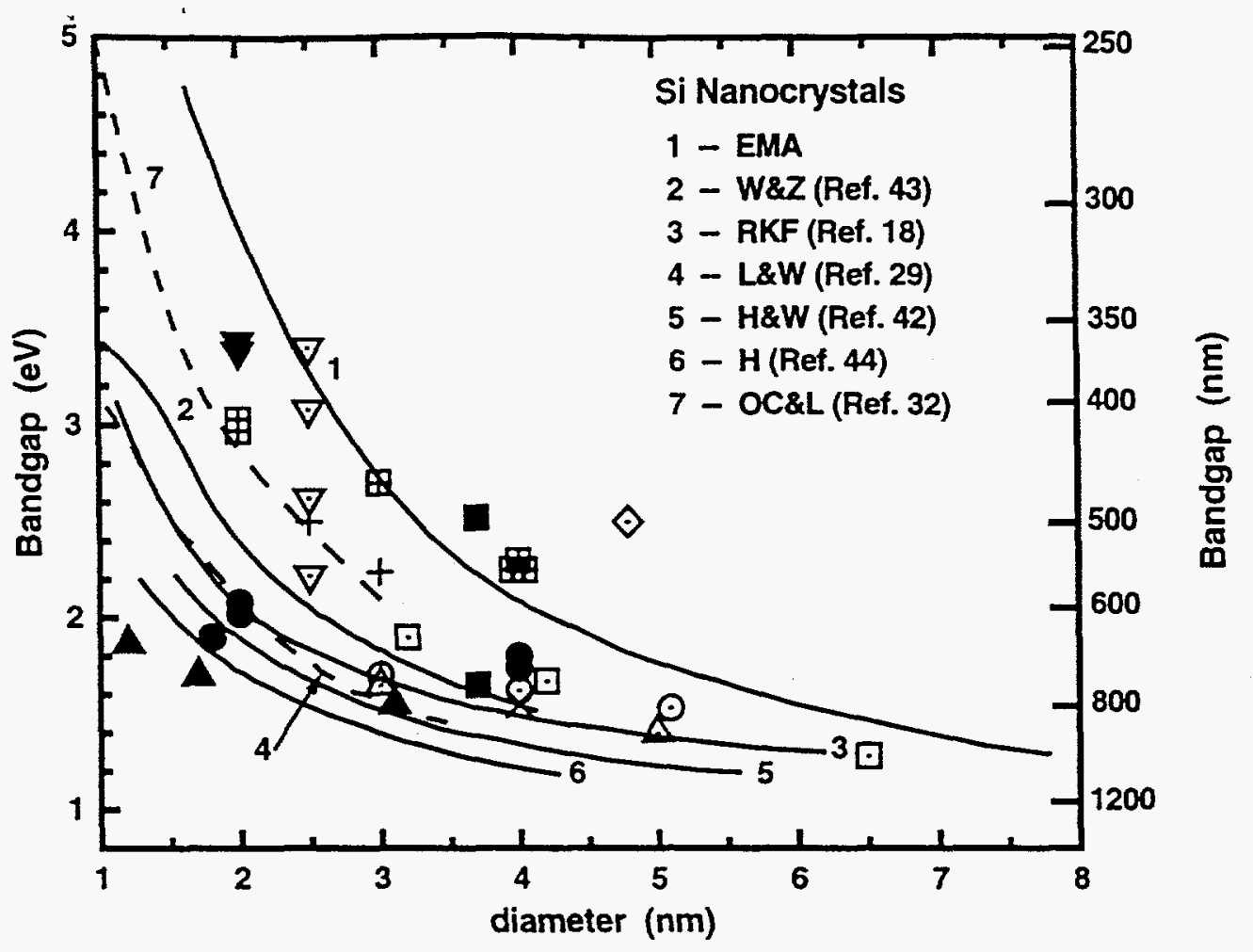

Figure 21

Wilcoxon et al

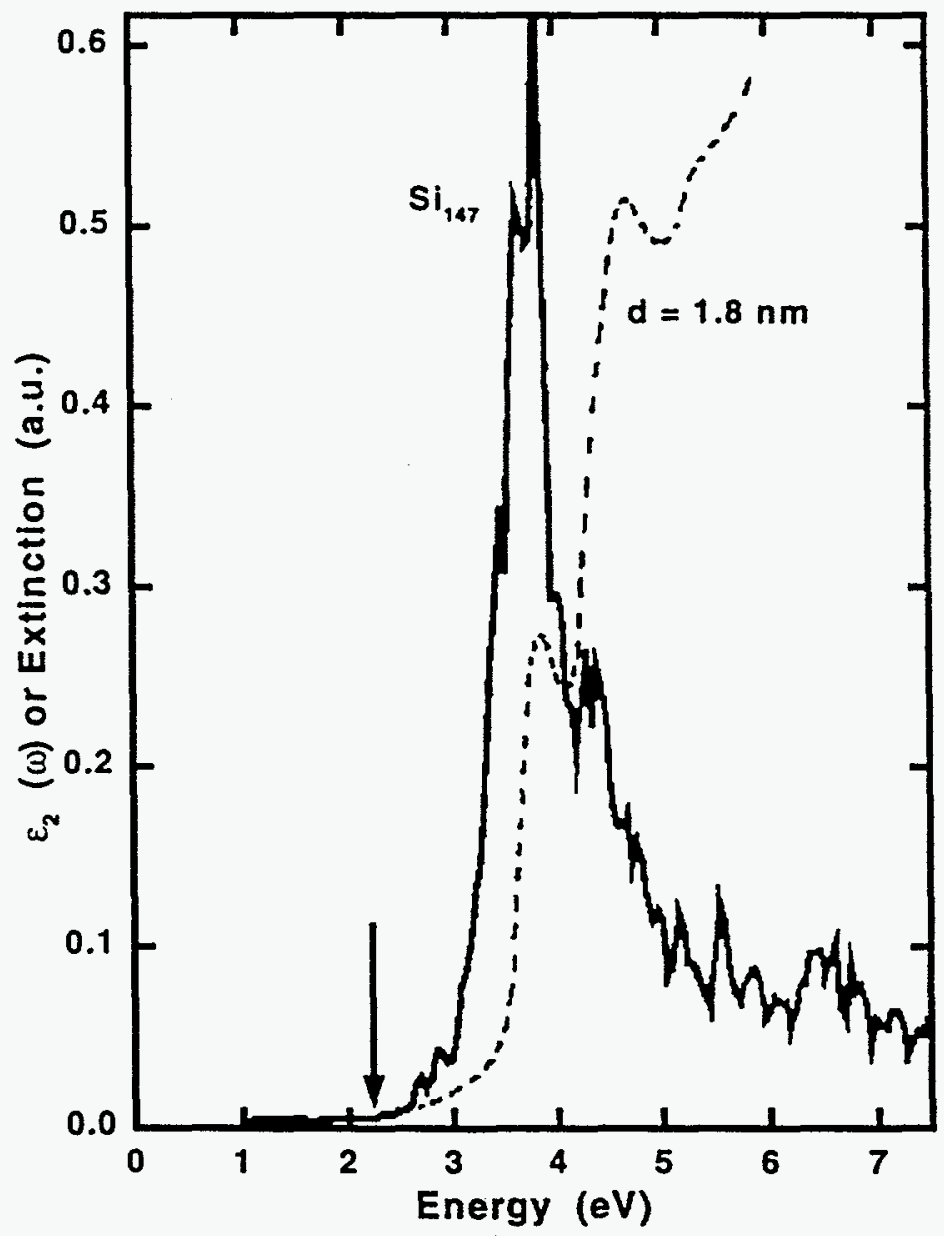

Figure 22

Wilcoxon et al 


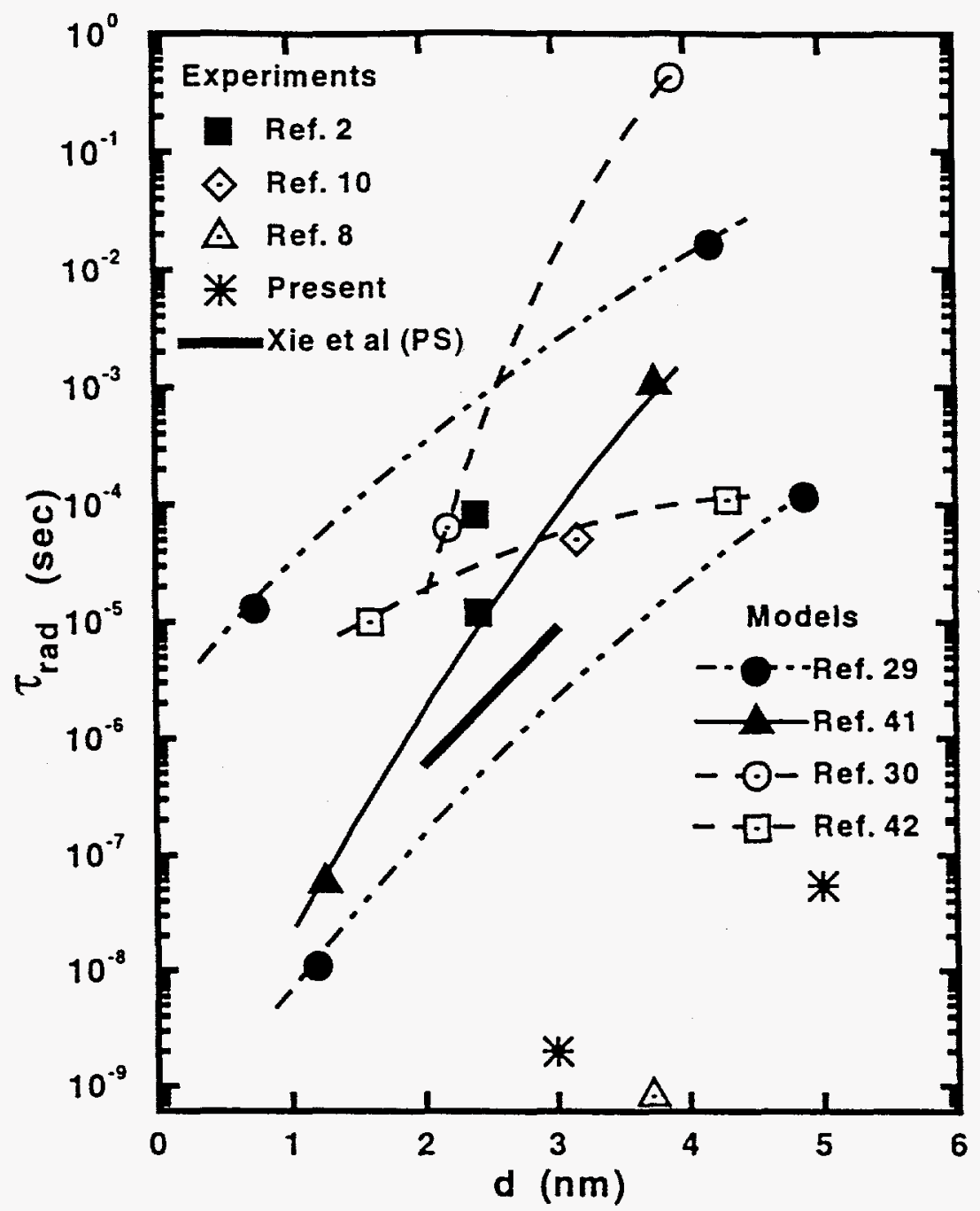

Figure 23

Wilcoxon et al 